\title{
Parity, hormones and breast cancer subtypes - results from a large nested case-control study in a national screening program
}

Merete Ellingjord-Dale ${ }^{1}$ D, Linda Vos ${ }^{1}$, Steinar Tretli ${ }^{1}$, Solveig Hofvind ${ }^{1}$, Isabel dos-Santos-Silva ${ }^{2}$ and Giske Ursin ${ }^{1,3,4^{*}}$

\begin{abstract}
Background: Breast cancer comprises several molecular subtypes with different prognoses and possibly different etiology. Reproductive and hormonal factors are associated with breast cancer overall, and with luminal subtypes, but the associations with other subtypes are unclear. We used data from a national screening program to conduct a large nested case-control study.

Methods: We conducted a nested case-control study on participants in the Norwegian Breast Cancer Screening Program in 2006 - 2014. There was information on estrogen receptor (ER), progesterone receptor (PR) and human epidermal growth factor receptor 2 (HER2) for 4748 cases of breast cancer. Breast cancer subtypes were defined as luminal A-like (ER+ PR+ HER2-), luminal B-like (ER+ PR- HER2- or ER+ PR+/PR-HER2+), HER2-positive (ER- PR- HER2+) and triple-negative (ER- PR- HER2-). Conditional logistic regression was used to estimate odds ratios (ORs) of breast cancer associated with age at first birth, number of pregnancies, oral contraceptive use, intrauterine devices and menopausal hormone therapy. Analyses were adjusted for age, body mass index, education, age at menarche, number of pregnancies and menopausal status.
\end{abstract}

Results: Number of pregnancies was inversely associated with relative risk of luminal-like breast cancers ( $p$-trend $\leq 0.02)$, and although not statistically significant, with HER2-positive $(\mathrm{OR}=0.60,95 \% \mathrm{Cl} 0.31-1.19)$ and triple-negative cancer ( $\mathrm{OR}=0.70,95 \% \mathrm{Cl} 0.41-1.21)$. Women who had $\geq 4$ pregnancies were at $>40 \%$ lower risk of luminal-like and HER2-positive cancers than women who had never been pregnant. However, there was a larger discrepancy between tumor subtypes with menopausal hormone use. Women who used estrogen and progesterone therapy (EPT) had almost threefold increased risk of luminal A-like cancer ( $\mathrm{OR}=2.92,95 \% \mathrm{Cl} 2.36-3.62)$ compared to neverusers, but were not at elevated risk of HER2-positive $(\mathrm{OR}=0.88,95 \% \mathrm{Cl} 0.33-2.30)$ or triple-negative $(\mathrm{OR}=0.92,95 \%$ Cl $0.43-1.98)$ subtypes.

Conclusions: Reproductive factors were to some extent associated with all subtypes; the strongest trends were with luminal-like subtypes. Hormone therapy use was strongly associated with risk of luminal-like breast cancer, and less so with risk of HER2-positive or triple-negative cancer. There are clearly some, but possibly limited, etiologic differences between subtypes, with the greatest contrast between luminal A-like and triple-negative subtypes.

Trial registration: Not applicable.

Keywords: Reproductive factors, Hormone therapy, Breast cancer subtypes

\footnotetext{
* Correspondence: Giske.Ursin@kreftregisteret.no

${ }^{1}$ Cancer Registry of Norway, Oslo, Norway

3University of Oslo, Oslo, Norway

Full list of author information is available at the end of the article
} 


\section{Background}

There is substantial evidence for a role of female hormones in the etiology of breast cancer. Reproductive factors, such as early age at menarche, nulliparity, and late age at first birth [1-3] are all believed to be associated with breast cancer risk through hormonal mechanisms. Current use of oral contraceptives is associated with some increased risk of breast cancer [4-6]. Likewise, combined postmenopausal hormone therapy (estrogen and progesterone), increases the risk of breast cancer [7-16]. However, breast cancer consists of several molecular subtypes that have very different prognoses [17-23]. It is less clear whether these various subtypes have different etiologies. There have been two main challenges in previous literature: the various definitions used to define breast cancer subtypes, and the lack of power to examine the effect on all subtypes.

In earlier studies, subtypes were defined by immunohistochemical analysis (IHC) of the two hormone receptors only, i.e. the estrogen receptor (ER) and the progesterone receptor (PR) [24-31]. It is only in recent years that studies have been conducted incorporating data on human epidermal growth factor receptor 2 (HER2) status [32-38], with a few having included other additional molecular markers [3, 34, 39-44]. However, most studies so far have not been able to run molecular expression studies on a large scale. This may not be necessary however, because there is a large degree of overlap between the immunohistochemical subtypes defined by ER, PR, and HER2 status and those identified by molecular expression studies [18-21, 45]. However, a challenge has been that investigators have used various definitions and specific markers to define breast cancer subtypes, such as luminal B tumors. Further, using the molecular terms luminal A, B, or basal gives the impression that they are defined using molecular expression markers, whereas they are based on IHC. Investigators at the St. Gallen meeting in 2013 therefore suggested that the term "like" should be added to the molecularly derived "luminal" subtype names (e.g. "luminal A-like", "luminal B-like") to indicate that IHC was used to define these subtypes [46] and hence, that they are proxies of the molecular subtypes.

Another challenge has been that many studies have not had adequate power to assess the effect of risk factors on all subtypes, which makes it difficult to determine the effect of risk factors on the less common subtypes. The overall published evidence $[7,8,10,47-50]$ seems to be consistent with luminal A-like cancers having a hormonal etiology, but the association between hormonal factors and other subtypes, in particular luminal B-like disease, HER2-positive disease, and triple-negative cancer, is less clear. Specifically, reproductive factors such as parity and early age at first birth have been associated with reduced risk of luminal A-like disease. There is less evidence of a protective effect of parity on luminal B-like and HER2-positive cancers and parity has consistently been found not to protect against triple-negative disease [7, 47-50]. There is some, albeit inconsistent, evidence that older age at menarche and breastfeeding may protect against all subtypes [7, 8, 47, 49-52] suggesting that these protective effects may work through non-hormonal mechanisms. The use of menopausal hormone therapy has been consistently associated with an increased risk of luminal A-like breast cancer, but the evidence is less clear for risk of luminal B-like, HER2-positive and triple-negative breast cancer [10, 47, 49].

We decided to take advantage of a national screening program in Norway to examine potential associations between reproductive and hormonal factors and the various breast cancer subtypes - specifically, luminal A-like, luminal B-like HER2-negative, luminal B-like HER2-positive, HER2-positive, and triple-negative disease - in a large study. We therefore conducted a large nested case-control study within the Norwegian Breast Cancer Screening Program.

\section{Methods}

\section{Study population}

The Cancer Registry of Norway (CRN) is responsible for the administration of the Norwegian Breast Cancer Screening Program [53]. Women aged 50-69 years are invited to undergo two-view mammography screening every 2 years. From August 2006, women, who underwent mammographic screening in the national program were asked to complete a questionnaire on a number of standard breast cancer risk factors, and another questionnaire on current exposure variables at subsequent screenings. For the current study cohort, women who had participated in the Norwegian Breast Cancer Screening Program during 2006 to 2014 and had completed these questionnaires were eligible for inclusion. The study cohort comprised a total of 344,348 eligible women. Information on cancer cases was obtained through linkage to the population-based CRN records using the unique 11-digit personal identification number assigned to all residents at time of birth. This linkage also included information on vital status, including date and cause of death/date of emigration if applicable. Reporting of cancer to the CRN is 
mandatory by law, and the registry is considered to be $98.8 \%$ complete [54].

All women aged 50-69 years who are included in the National Population Registry are invited to undergo screening as part of the Norwegian Breast Cancer Screening Program every 2 years. The average attendance rate in each round is about $76 \%$.

We conducted a nested case-control study within the study cohort. Only women with no history of ductal carcinoma in situ prior to the study start (1 January 2006) and no history of diagnosis of another invasive cancer (except non-melanoma skin cancer) were eligible for the study. The cases were women diagnosed with a first occurrence of invasive breast cancer (ICD10: C50) during the study period, with information on ER, PR, and HER2 status (see subsequent text). For each woman with breast cancer, we randomly selected five controls individually matched to cases by year of birth $(+/-3$ years $)$ and year of last screening before breast cancer diagnosis (+/3 years). Controls had to be alive and resident in the country at the time of the diagnosis of breast cancer in the matching case. We ended up with 6471 patients with breast cancer (cases) and 32,355 controls. The study was approved by the Regional Committee for Medical and Health Research Ethics in the South-East Health Region of Norway.

\section{Tumor receptor status ascertainment}

Information on ER, PR and HER2 status was assessed by IHC and extracted from pathology reports submitted to the CRN. From 2006 to January 2012, tumors were classified as ER-negative if there was $<10 \%$ reactivity. From February 2012 onwards, the threshold for ER-negative tumors was changed to $<1 \%$ reactivity as a result of change in the treatment protocols for patients attending clinics in Norway. We used these official thresholds. PR-negative tumors were defined as those with reactivity of $<10 \%$, and PRpositive tumors as those with reactivity $\geq 10 \%$. HER 2 expression status was determined at each laboratory with IHC and/or in situ hybridization. Cases with no (0) or weak $(1+)$ immunostaining were defined as HER2-negative, while cases with moderate $(2+)$ or strong immunostaining $(3+)$ were defined as HER2positive. In situ hybridization (fluorescence (FISH), chromogenic (CISH), or silver (SISH) in situ hybridization methods) was usually used to confirm HER2 status if IHC yielded 2+ results. If IHC was 2+ and FISH, CISH, or SISH were missing, or if IHC was missing but FISH, CISH, or SISH were positive, the tumor was classified as HER2-positive. If IHC was $2+$ and FISH, CISH, and SISH were negative, the tumor was regarded as HER2-negative. Data on Ki-67 were recorded by the CRN from late 2011 and therefore, we did not include this marker in our analysis.

\section{Risk factors}

Our primary exposures of interest were hormonal risk factors including reproductive factors (age at first birth, number of pregnancies, breastfeeding, menopausal status), and other hormonal factors (use of oral contraceptives, intrauterine devices (IUD) and menopausal hormone therapy use). Menopausal hormone therapy use included use of estrogen alone (estrogen therapy, ET), or use of combined estrogen and progestin therapy (EPT). For current exposures we chose the questionnaire at the last screening before breast cancer diagnosis for the cases and the corresponding time for the controls. If the questionnaire or any values were missing, information was used from the previous screening questionnaire. Menopausal status was defined according to whether or not women were still menstruating, or whether they menstruated irregularly. Menopausal age was defined as the age when menstruation ended.

\section{Confounders and missing values}

We considered age at screening $(50-54,55-59,60-$ 64 , or 65-70 years), body mass index (BMI) at screening ( $\leq 22,23-25,26-28$, or $>28$ ), education (no education/primary school, high school, or bachelor's/ master's degree), age at menarche $(9-12,13,14$, or 15-18 years), number of pregnancies lasting at least six months (never pregnant, 1, 2, 3, or $\geq 4$ ) and menopausal status (premenopausal, perimenopausal, or postmenopausal) as potential confounders and adjusted for these when appropriate. We tried to control more tightly for age at screening using a continuous variable, but the results remained largely the same, and we therefore retained the 5-year categories.

Women with missing values for an exposure variable were excluded from the analyses of that variable, while women with missing information on the potential confounding variables listed previously were excluded from all analyses. Of the $6471 \mathrm{pa}$ tients with breast cancer, we excluded women due to missing information on the following variables: BMI ( $n=532$ patients), age at menarche $(n=298$ patients), education ( $n=66$ patients), number of pregnancies ( $n=164$ patients), and menopausal status $(n=59$ patients). This left us with 5352 women with breast cancer (cases) for analysis. Of the 32,355 controls, we excluded controls based on missing information on the following: BMI ( $n=3296$ controls), age at 
menarche $(n=1641$ controls $)$, education $(n=371$ controls), number of pregnancies $(n=779$ controls), and menopausal status ( $n=336$ controls). This left us with 25,932 controls for analysis.

The cases were categorized by breast cancer subtype using a modified version of the classification of clinically defined subtypes proposed at the St. Gallen meeting in 2013 [46]. Of the 5352 cases of breast cancer, 604 had unknown hormone receptor (i.e. ER and/or PR) and HER2 status or could not be classified into the breast cancer subtypes. There were 4748 women with breast cancer classified into the following subtypes: 2985 women with luminal A-like breast cancer (ER+ PR+ HER2-), 758 women with luminal B-like HER2-negative breast cancer (ER+ PR- HER2-), 396 women with luminal B-like HER2positive breast cancer (ER+ PR+/PR- HER2+), 223 women with HER2-positive breast cancer (ER- PRHER2+) and 386 women with triple-negative breast cancer (ER- PR- HER2-). As we did not have Ki-67 results, we conducted sensitivity analysis where we added grade to the luminal subtype definitions in an attempt to better separate out these subtypes, using the definitions from the St. Gallen 2013 meeting [46]. In this analysis luminal A-like subtype was defined as ER+ PR+ HER2-, low or medium grade, luminal B-like HER2-negative as ER+ PR- HER2-, high grade, and luminal B-like HER2-positive as ER+ PR-/ $\mathrm{PR}+\mathrm{HER} 2+$, any grade.

\section{Statistical analyses}

We used conditional logistic regression to estimate odds ratios (with 95\% confidence intervals $(\mathrm{CI})$ ) as a measure of relative risk associated with various exposures. Trend tests were performed by fitting ordinal values corresponding to exposure categories and testing whether the slope coefficient differed from zero. To test for heterogeneity between breast cancer subtypes we ran case-case analyses, comparing each subtype to the luminal-A-like subtype. We used likelihood ratio tests comparing the likelihood ratio of the case-case model with confounders only to that of the adjusted case-case model with the exposure variable. We considered a two-sided $p$ value $<0.05$ as statistically significant.

Because we used conditional logistic regression we included all women in the risk estimates (e.g. women who were never pregnant were included in analysis of age at first birth, women who never used oral contraceptives (OC) were included in analysis of age at the start of OC, and premenopausal women were included in analysis of age at menopause), but they were not included in the trend test (dummy variables were added into the analysis).

\section{Results}

BMI, age at first birth, education, age at menopause, duration of use of oral contraceptives and intrauterine devices, and menopausal hormone therapy use were positively associated with overall breast cancer risk (i.e. all subtypes combined) whereas age at menarche, number of pregnancies and postmenopausal status were associated with a decreased risk (Table 1).

Number of pregnancies was inversely associated with risk of breast cancer overall and risk of several breast cancer subtypes (Table 2). Compared to women who had never been pregnant, those with $\geq 4$ pregnancies had about $40 \%$ or lower risk of developing luminal-like breast cancer, and all three tests for trend were statistically significant (Table 2). There were similar, but non-significant protective associations also with HER2-positive and triple-negative tumors, but neither the point estimate for the top category $(\geq 4$ pregnancies: $\mathrm{OR}=0.60,95 \%$ CI $0.31-$ 1.19 and $\mathrm{OR}=0.70,95 \% \mathrm{CI} 0.41-1.21$, respectively), nor the trend for number of pregnancies was statistically significant. The test for heterogeneity comparing triple-negative cancer to luminal A-like cancer was statistically significant.

Later age at first birth was associated with an increased risk of breast cancer overall and with several breast cancer subtypes (Table 2). Compared to those with an early first birth ( $\leq 20$ years), women with a later age at first birth ( $>30$ years) were at slightly increased risk of luminal A-like $(\mathrm{OR}=1.19,95 \% \mathrm{CI}$ 0.99-1.43) and luminal B-like HER2-negative breast cancer $(\mathrm{OR}=1.60,95 \%$ CI $1.10-2.32, p$-trend $=0.07$ and 0.01 , respectively). The ORs associated with the oldest age at first birth ( $>30$ years) were also similarly elevated, but not significantly so, for HER2-positive $(\mathrm{OR}=1.67,95 \%$ CI $0.89-3.12)$ and triple-negative $(\mathrm{OR}=1.47,95 \%$ CI $0.88-2.47)$ subtypes $(p$-trend $=$ 0.49 and 0.16, respectively). However, the test for heterogeneity comparing each subtype to luminal Alike breast cancer was not statistically significant for age at first birth.

Duration of breastfeeding was not statistically significantly associated with breast cancer overall ( $p$ trend $=0.14$ ) (Table 1 ) or with any of the subtypes (Table 2). Postmenopausal status was associated with a statistically significantly reduced risk of breast cancer overall (Table 1) and of luminal A-like breast cancer compared to being premenopausal $(\mathrm{OR}=0.51$, 95\% CI 0.43-0.60), while no association was observed for any of the other subtypes, and all tests for heterogeneity were statistically significant. Age at menopause, comparing women $>52$ to women $<47$ years old was positively associated with risk of luminal Blike HER2-positive $(\mathrm{OR}=1.70,95 \% \mathrm{CI} 1.13-2.55, p$ - 
Table 1 Adjusted odds ratios (ORs) and 95\% confidence intervals (Cl) for association between breast cancer overall and education and reproductive and hormonal risk factors

\begin{tabular}{|c|c|c|c|c|c|}
\hline \multirow[t]{2}{*}{ Characteristics } & \multicolumn{5}{|l|}{ Overall } \\
\hline & Total $(n)$ & Controls $(n)$ & Cases (n) & $\mathrm{OR}^{*}$ & $95 \% \mathrm{Cl}$ \\
\hline \multicolumn{6}{|l|}{ Education } \\
\hline No education/primary school & 8584 & 7192 & 1392 & 1 & Ref \\
\hline High school & 12,781 & 10,582 & 2199 & 1.07 & $(0.99,1.15)$ \\
\hline University Bachelor's degree & 6179 & 5081 & 1098 & 1.11 & $(1.01,1.22)$ \\
\hline University Master's degree & 3740 & 3077 & 663 & 1.10 & $(0.98,1.22)$ \\
\hline p-trend* & & & & 0.03 & \\
\hline \multicolumn{6}{|l|}{ BMI $\left(\mathrm{kg} / \mathrm{m}^{2}\right)$} \\
\hline$<=22$ & 5141 & 4333 & 808 & 1 & Reference \\
\hline $23-25$ & 9655 & 8075 & 1580 & 1.07 & $(0.98,1.18)$ \\
\hline $26-28$ & 8131 & 6694 & 1437 & 1.19 & $(1.08,1.32)$ \\
\hline$>28$ & 8357 & 6830 & 1527 & 1.23 & $(1.12,1.36)$ \\
\hline$p$-trend & & & & $<0.0001$ & \\
\hline \multicolumn{6}{|l|}{ Age at menarche (years) } \\
\hline $9-12$ & 9296 & 7615 & 1681 & 1 & Reference \\
\hline 13 & 8628 & 7157 & 1471 & 0.93 & $(0.86,1.01)$ \\
\hline 14 & 7704 & 6439 & 1265 & 0.89 & $(0.82,0.97)$ \\
\hline $15-18$ & 5656 & 4721 & 935 & 0.90 & $(0.82,0.98)$ \\
\hline$p$-trend & & & & 0.01 & \\
\hline \multicolumn{6}{|l|}{ Age at first birth (years) } \\
\hline $13-20$ & 8413 & 7085 & 1328 & 1 & Reference \\
\hline $21-22$ & 4994 & 4170 & 824 & 1.04 & $(0.94,1.15)$ \\
\hline $23-25$ & 6673 & 5580 & 1093 & 1.02 & $(0.93,1.12)$ \\
\hline $26-30$ & 5440 & 4497 & 943 & 1.09 & $(0.98,1.20)$ \\
\hline $31-50$ & 2070 & 1641 & 429 & 1.29 & $(1.12,1.48)$ \\
\hline Never given birth (nulliparous) & 2730 & 2144 & 586 & 1.73 & $(1.49,2.01)$ \\
\hline$p$-trend ${ }^{a}$ & & & & 0.003 & \\
\hline \multicolumn{6}{|c|}{ Number of pregnancies lasting $6+$ months } \\
\hline 0 & 2730 & 2144 & 586 & 1 & Reference \\
\hline 1 & 3584 & 2911 & 673 & 0.83 & $(0.73,0.94)$ \\
\hline 2 & 13499 & 11000 & 2301 & 0.76 & $(0.68,0.84)$ \\
\hline 3 & 8307 & 6956 & 1351 & 0.71 & $(0.63,0.79)$ \\
\hline$>4$ & 3164 & 2723 & 441 & 0.59 & $(0.51,0.68)$ \\
\hline$p$-trend & & & & $<0.0001$ & \\
\hline \multicolumn{6}{|l|}{ Parous women only } \\
\hline \multicolumn{6}{|c|}{ Duration of breastfeeding (months) } \\
\hline Parous no breastfeeding & 2201 & 1840 & 361 & 1 & Reference \\
\hline $1-6$ & 6872 & 5775 & 1097 & 0.97 & $(0.84,1.11)$ \\
\hline $7-12$ & 6662 & 5452 & 1210 & 1.16 & $(1.01,1.34)$ \\
\hline $13-20$ & 5466 & 4544 & 922 & 1.08 & $(0.93,1.25)$ \\
\hline $21-30$ & 3620 & 3043 & 577 & 1.04 & $(0.88,1.22)$ \\
\hline$>30$ & 2067 & 1736 & 331 & 1.10 & $(0.91,1.33)$ \\
\hline$p$-trend & & & & 0.14 & \\
\hline
\end{tabular}


Table 1 Adjusted odds ratios (ORs) and 95\% confidence intervals (Cl) for association between breast cancer overall and education and reproductive and hormonal risk factors (Continued)

\begin{tabular}{|c|c|c|c|c|c|}
\hline \multicolumn{6}{|l|}{ Menopausal status } \\
\hline Premenopausal & 2568 & 2035 & 533 & 1 & Reference \\
\hline Perimenopausal & 2279 & 1876 & 403 & 0.73 & $(0.63,0.86)$ \\
\hline Postmenopausal & 26437 & 22000 & 4416 & 0.66 & $(0.59,0.75)$ \\
\hline$p$-trend & & & & $<0.0001$ & \\
\hline \multicolumn{6}{|c|}{ Age at menopause (years) } \\
\hline$<47$ & 5412 & 4595 & 817 & 1 & Reference \\
\hline $47-49$ & 5151 & 4324 & 827 & 1.10 & $(0.99,1.22)$ \\
\hline 50-52 & 9831 & 8218 & 1613 & 1.13 & $(1.03,1.24)$ \\
\hline$>52$ & 6663 & 5508 & 1155 & 1.15 & $(1.04,1.28)$ \\
\hline Premenopausal & 2568 & 2035 & 533 & 1.81 & $(1.57,2.10)$ \\
\hline$p$-trend $^{\mathrm{b}}$ & & & & 0.01 & \\
\hline \multicolumn{6}{|c|}{ Age at start of oral contraceptives (years) } \\
\hline $14-18$ & 3097 & 2577 & 520 & 1 & Reference \\
\hline $19-20$ & 3356 & 2686 & 670 & 1.19 & $(1.02,1.38)$ \\
\hline $21-24$ & 4190 & 3490 & 700 & 0.95 & $(0.82,1.11)$ \\
\hline $25-50$ & 4307 & 3590 & 717 & 0.91 & $(0.77,1.07)$ \\
\hline Never used & 14532 & 12000 & 2443 & 0.95 & $(0.85,1.07)$ \\
\hline p-trend ${ }^{a}$ & & & & 0.06 & \\
\hline \multicolumn{6}{|c|}{ Duration of oral contraceptives (years) } \\
\hline Never used & 14532 & 12000 & 2443 & 1 & Reference \\
\hline$<2$ & 4019 & 3405 & 614 & 0.89 & $(0.81,0.99)$ \\
\hline $2-5$ & 3758 & 3120 & 638 & 1.02 & $(0.92,1.13)$ \\
\hline $6-10$ & 3453 & 2834 & 619 & 1.10 & $(0.99,1.22)$ \\
\hline$>10$ & 2718 & 2203 & 515 & 1.11 & $(1.00,1.25)$ \\
\hline$p$-trend & & & & 0.01 & \\
\hline \multicolumn{6}{|c|}{ Age at start of intrauterine device (years) } \\
\hline $14-28$ & 1340 & 1120 & 220 & 1 & Reference \\
\hline $29-35$ & 1422 & 1174 & 248 & 1.03 & $(0.84,1.27)$ \\
\hline $36-42$ & 1193 & 965 & 228 & 1.18 & $(0.95,1.46)$ \\
\hline $43-50$ & 1052 & 859 & 193 & 1.06 & $(0.85,1.33)$ \\
\hline Never used & 21734 & 18000 & 3693 & 0.99 & $(0.84,1.16)$ \\
\hline p-trend ${ }^{a}$ & & & & 0.27 & \\
\hline \multicolumn{6}{|c|}{ Duration of intrauterine device (years) } \\
\hline Never used & 21734 & 18000 & 3693 & 1 & Reference \\
\hline$<2$ & 729 & 612 & 117 & 0.92 & $(0.75,1.14)$ \\
\hline $2-5$ & 969 & 791 & 178 & 1.07 & $(0.90,1.28)$ \\
\hline $6-10$ & 1403 & 1158 & 245 & 1.10 & $(0.95,1.28)$ \\
\hline$>10$ & 1753 & 1432 & 321 & 1.14 & $(0.99,1.30)$ \\
\hline$p$-trend & & & & 0.03 & \\
\hline \multicolumn{6}{|c|}{ Hormone therapy use } \\
\hline Never & 15688 & 13000 & 2487 & 1 & Reference \\
\hline Past & 10548 & 8656 & 1892 & 1.16 & $(1.08,1.25)$ \\
\hline
\end{tabular}


Table 1 Adjusted odds ratios (ORs) and 95\% confidence intervals (Cl) for association between breast cancer overall and education and reproductive and hormonal risk factors (Continued)

\begin{tabular}{|c|c|c|c|c|c|}
\hline Estrogen current & 1468 & 1219 & 249 & 1.03 & $(0.88,1.20)$ \\
\hline Estrogen and progesterone current & 953 & 661 & 292 & 2.32 & $(1.97,2.72)$ \\
\hline$p$-trend & & & & $<0.0001$ & \\
\hline \multicolumn{6}{|l|}{ Duration of hormone therapy (years) } \\
\hline Never used & 15688 & 13000 & 2487 & 1 & Reference \\
\hline$<=3$ & 3636 & 3066 & 570 & 1.00 & $(0.90,1.10)$ \\
\hline $4-8$ & 2449 & 2013 & 436 & 1.17 & $(1.03,1.30)$ \\
\hline$>8$ & 4060 & 3151 & 909 & 1.58 & $(1.42,1.70)$ \\
\hline$p$-trend & & & & $<0.0001$ & \\
\hline \multicolumn{6}{|c|}{ Duration of estrogen and progesterone therapy (years) } \\
\hline Never used & 15688 & 13000 & 2487 & 1 & Reference \\
\hline$<=5$ & 4288 & 3552 & 736 & 1.13 & $(1.02,1.25)$ \\
\hline$>5$ & 4189 & 3238 & 951 & 1.61 & $(1.46,1.78)$ \\
\hline$p$-trend & & & & $<0.0001$ & \\
\hline
\end{tabular}

${ }^{a}$ The category Never used was not included in the analysis of $p$-trend. bThe category Premenopausal was not included in the analysis of $p$-trend. ${ }^{*} p$ for trend and OR mutually adjusted for age $(50-54,55-59,60-64,65-70$ years at screening), body mass index (BMI) $(\leq 22,23-25,26-28,>28$ at screening), education (no education/primary school, high school, bachelor's or master's degree), age at menarche $(9-12,13,14,15-18$ years), number of pregnancies (0, 1, 2, 3, $\geq 4)$, menopausal status (premenopausal, perimenopausal, postmenopausal)

trend $=0.01)$ and HER2-positive $(\mathrm{OR}=2.36,95 \% \mathrm{CI}$ $1.33-4.21, p$-trend $=0.004$ ) subtypes (Table 2).

Duration of oral contraceptive use and use of an IUD, were associated with a slight increased risk of breast cancer overall (Table 1), with $10 \%$ elevated ORs among women with the longest duration of use. The effect estimates for long duration of oral contraceptive use were similar, but not statistically significant, for luminal A-like $(\mathrm{OR}=1.11,95 \%$ CI $0.96-$ 1.29) and luminal B-like HER2-negative ( $\mathrm{OR}=1.12$, 95\% CI 0.81-1.54) subtypes. Women who had used an IUD for more than 10 years had an increased risk of luminal A-like breast cancer compared to never users $(\mathrm{OR}=1.35,95 \%$ CI 1.14-1.61) (Table 3). We observed no evidence of any clear associations for the other subtypes, and in fact a reduced OR for the luminal B-like HER2-positive $(\mathrm{OR}=0.51,95 \% \mathrm{CI}$ $0.27-0.96$ ) subtype ( $p$-heterogeneity with luminal Alike breast cancer $=0.009)$ (Table 3$)$.

Compared to women who had never used EPT, current use of EPT was associated with an increased risk $(\mathrm{OR}=2.32,95 \% \mathrm{CI} 1.97-2.72)$ of breast cancer overall (Table 1) and an increased risk of luminal Alike breast cancer $(\mathrm{OR}=2.92,95 \%$ CI 2.36-3.62) (Table 3). The ORs for both luminal B-like subtypes were about 1.7 , but this was only statistically significant for the luminal B-like HER2-negative subtype. There was no increased risk of triple-negative breast cancer with the use of EPT ( $p$-heterogeneity $=0.006)$. Previous studies have suggested that the effect of menopausal hormone therapy may be modified by
BMI, with stronger risk estimates in lean than in obese women. We therefore examined the effect of duration of EPT in different BMI strata. For breast cancer overall, the ORs associated with longer duration of EPT was significantly higher in lean (BMI $<25)$ than in obese (BMI $\geq 25)$ women ( $p$ for interaction $=0.001) \quad($ Table 4$)$. We observed similar effect modifications by BMI in luminal A-like and luminal B-like HER2-negative breast cancer. There was no evidence that BMI modified the associations between duration of EPT and risk of any of the other subtypes (Table 4).

When we added grade to the luminal A-like and both luminal B-like definitions, the results changed slightly, but were largely the same (Appendix). The largest difference was a slightly stronger effect of current EPT on the luminal A-like subtype $(O R=3.03$ when grade was included in the definition versus $\mathrm{OR}=2.90$ when it was not).

Among the patients (cases), 38\% $(n=1813)$ were diagnosed within a month of completing the questionnaire. Because these women could have been symptomatic when they completed their questionnaire, we ran a sensitivity analysis excluding these women. However, this did not affect the results (results not shown).

\section{Discussion}

In this population-based study within a national screening program, we had information on 4748 patients with breast cancer, which makes it one of the 
Table 2 Adjusted odds ratios (OR) and 95\% confidence intervals (CI) for association between breast cancer subtypes associated and reproductive factors

\begin{tabular}{|c|c|c|c|c|c|c|c|c|c|c|c|c|c|c|c|c|c|c|c|c|}
\hline & \multicolumn{4}{|c|}{$\begin{array}{l}\text { Luminal A-like } \\
\text { ER+PR+ HER2- }\end{array}$} & \multicolumn{4}{|c|}{$\begin{array}{l}\text { Luminal B-like HER2-negative } \\
\text { ER+ PR- HER2- } \\
\end{array}$} & \multicolumn{4}{|c|}{$\begin{array}{l}\text { Luminal B-like HER2-positive } \\
\text { ER+ PR+/PR- HER2+- }\end{array}$} & \multicolumn{4}{|c|}{$\begin{array}{l}\text { HER2-positive } \\
\text { ER- PR- HER2+ } \\
\end{array}$} & \multicolumn{4}{|c|}{$\begin{array}{l}\text { Triple-negative } \\
\text { ER- PR- HER2- } \\
\end{array}$} \\
\hline & $\begin{array}{l}\text { Controls } \\
(n)\end{array}$ & $\begin{array}{l}\text { Cases } \\
(n)\end{array}$ & $O R^{*}$ & $95 \% \mathrm{Cl}$ & $\begin{array}{l}\text { Controls } \\
(n)\end{array}$ & $\begin{array}{l}\text { Cases } \\
(n)\end{array}$ & OR & $95 \% \mathrm{Cl}$ & $\begin{array}{l}\text { Controls } \\
(n)\end{array}$ & $\begin{array}{l}\text { Cases } \\
(n)\end{array}$ & OR & $95 \% \mathrm{Cl}$ & Controls $(n)$ & $\begin{array}{l}\text { Cases } \\
(n)\end{array}$ & OR & $95 \% \mathrm{Cl}$ & $\begin{array}{l}\text { Controls } \\
(n)\end{array}$ & $\begin{array}{l}\text { Cases } \\
(n)\end{array}$ & OR & $95 \% \mathrm{Cl}$ \\
\hline \multicolumn{21}{|l|}{ Age at first birth (years) } \\
\hline $13-20$ & 3951 & 746 & 1 & Reference & 1001 & 169 & 1 & Reference & 553 & 96 & 1 & Reference & 279 & 54 & 1 & Reference & 495 & 95 & 1 & Reference \\
\hline $21-22$ & 2277 & 460 & 1.04 & $(0.91,1.18)$ & 620 & 120 & 1.22 & $(0.93,1.59)$ & 305 & 51 & 0.97 & $(0.65,1.43)$ & 170 & 32 & 0.97 & $(0.58,1.62)$ & 326 & 65 & 1.10 & $(0.77,1.57)$ \\
\hline $23-25$ & 3152 & 591 & 0.97 & $(0.85,1.10)$ & 759 & 157 & 1.29 & $(1.00,1.67)$ & 437 & 92 & 1.27 & $(0.90,1.78)$ & 222 & 36 & 0.75 & $(0.46,1.22)$ & 393 & 86 & 1.27 & $(0.89,1.81)$ \\
\hline $26-30$ & 2473 & 547 & 1.12 & $(0.98,1.28)$ & 647 & 141 & 1.30 & $(0.99,1.71)$ & 343 & 62 & 1.21 & $(0.82,1.78)$ & 216 & 41 & 0.97 & $(0.59,1.58)$ & 318 & 63 & 1.16 & $(0.79,1.70)$ \\
\hline $31-50$ & 937 & 238 & 1.19 & $(0.99,1.43)$ & 223 & 60 & 1.60 & $(1.10,2.32)$ & 127 & 32 & 1.64 & $(0.98,2.74)$ & 63 & 23 & 1.67 & $(0.89,3.12)$ & 120 & 29 & 1.47 & $(0.88,2.47)$ \\
\hline Never (nulliparous) & 1199 & 328 & 1.76 & $(1.44,2.15)$ & 303 & 89 & 1.96 & $(1.31,2.91)$ & 164 & 49 & 2.74 & $(1.57,4.78)$ & 84 & 27 & 1.82 & $(0.85,3.88)$ & 144 & 34 & 1.58 & $(0.89,2.81)$ \\
\hline$p$-trend ${ }^{a}$ & & & 0.07 & & & & 0.01 & & & & 0.06 & & & & 0.49 & & & & 0.16 & \\
\hline p-heterogeneity ${ }^{\#}$ & & & & & & & 0.19 & & & & 0.19 & & & & 0.31 & & & & 0.68 & \\
\hline \multicolumn{21}{|c|}{ Number of pregnancies lasting $6+$ months } \\
\hline 0 & 1199 & 328 & 1 & Reference & 303 & 89 & 1 & Reference & 164 & 49 & 1 & Reference & 84 & 27 & 1 & Reference & 144 & 34 & 1 & Reference \\
\hline 1 & 1596 & 397 & 0.91 & $(0.77,1.08)$ & 426 & 84 & 0.68 & $(0.48,0.96)$ & 229 & 42 & 0.53 & $(0.33,0.86)$ & 128 & 20 & 0.50 & $(0.26,0.96)$ & 224 & 41 & 0.70 & $(0.42,1.16)$ \\
\hline 2 & 6266 & 1302 & 0.76 & $(0.66,0.88)$ & 1602 & 323 & 0.68 & $(0.52,0.90)$ & 842 & 185 & 0.71 & $(0.49,1.04)$ & 426 & 97 & 0.78 & $(0.47,1.29)$ & 813 & 158 & 0.77 & $(0.50,1.18)$ \\
\hline 3 & 3883 & 721 & 0.66 & $(0.57,0.77)$ & 950 & 197 & 0.69 & $(0.52,0.93)$ & 528 & 89 & 0.54 & $(0.36,0.82)$ & 319 & 60 & 0.61 & $(0.36,1.03)$ & 477 & 117 & 0.98 & $(0.63,1.53)$ \\
\hline$>4$ & 1518 & 237 & 0.56 & $(0.46,0.68)$ & 383 & 65 & 0.58 & $(0.40,0.84)$ & 225 & 31 & 0.42 & $(0.25,0.70)$ & 98 & 19 & 0.60 & $(0.31,1.19)$ & 196 & 36 & 0.70 & $(0.41,1.21)$ \\
\hline$p$-trend & & & $<0.0001$ & & & & 0.02 & & & & 0.002 & & & & 0.19 & & & & 0.88 & \\
\hline$p$-heterogeneity & & & & & & & 0.31 & & & & 0.42 & & & & 0.34 & & & & 0.03 & \\
\hline \multicolumn{21}{|l|}{ Parous women only } \\
\hline \multicolumn{21}{|c|}{ Duration breastfeeding (months) } \\
\hline No breastfeeding & 997 & 204 & 1 & Reference & 260 & 51 & 1 & Reference & 146 & 33 & 1 & Reference & 78 & 10 & 1 & Reference & 135 & 24 & 1 & Reference \\
\hline $1-6$ & 3145 & 607 & 0.95 & $(0.79,1.15)$ & 831 & 170 & 1.01 & $(0.70,1.45)$ & 449 & 74 & 0.66 & $(0.40,1.07)$ & 243 & 37 & 1.42 & $(0.63,3.18)$ & 467 & 80 & 1.01 & $(0.60,1.71)$ \\
\hline $7-12$ & 3108 & 663 & 1.09 & $(0.91,1.31)$ & 763 & 158 & 1.05 & $(0.73,1.51)$ & 427 & 98 & 0.83 & $(0.51,1.33)$ & 215 & 52 & 2.25 & $(1.02,4.99)$ & 359 & 95 & 1.65 & $(0.98,2.78)$ \\
\hline $13-20$ & 2583 & 496 & 1.02 & $(0.83,1.24)$ & 654 & 130 & 0.98 & $(0.67,1.44)$ & 343 & 77 & 0.90 & $(0.54,1.49)$ & 156 & 48 & 2.51 & $(1.11,5.68)$ & 308 & 69 & 1.29 & $(0.74,2.23)$ \\
\hline $21-30$ & 1700 & 319 & 1.03 & $(0.83,1.28)$ & 399 & 79 & 0.97 & $(0.64,1.46)$ & 240 & 37 & 0.60 & $(0.33,1.10)$ & 147 & 28 & 1.78 & $(0.74,4.28)$ & 222 & 44 & 1.18 & $(0.65,2.15)$ \\
\hline$>30$ & 966 & 195 & 1.15 & $(0.89,1.48)$ & 250 & 48 & 1.03 & $(0.63,1.69)$ & 121 & 16 & 0.69 & $(0.33,1.47)$ & 70 & 13 & 1.82 & $(0.67,4.97)$ & 123 & 24 & 1.19 & $(0.58,2.44)$ \\
\hline$p$-trend & & & 0.22 & & & & 0.90 & & & & 0.63 & & & & 0.12 & & & & 0.52 & \\
\hline$p$-heterogeneity & & & & & & & 0.85 & & & & 0.25 & & & & 0.56 & & & & 0.84 & \\
\hline \multicolumn{21}{|l|}{ Menopausal status } \\
\hline Premenopausal & 1171 & 351 & 1 & Reference & 208 & 47 & 1 & Reference & 173 & 32 & 1 & Reference & 100 & 18 & 1 & Reference & 130 & 29 & 1 & Reference \\
\hline Perimenopausal & 1055 & 229 & 0.61 & $(0.50,0.74)$ & 225 & 50 & 0.95 & $(0.60,1.51)$ & 152 & 26 & 0.86 & $(0.47,1.54)$ & 79 & 20 & 1.41 & $(0.67,2.94)$ & 143 & 37 & 1.13 & $(0.64,1.99)$ \\
\hline Postmenopausal & 12000 & 2405 & 0.51 & $(0.43,0.60)$ & 3231 & 661 & 0.84 & $(0.58,1.23)$ & 1663 & 338 & 1.11 & $(0.69,1.78)$ & 876 & 185 & 1.21 & $(0.66,2.23)$ & 1581 & 320 & 0.82 & $(0.50,1.33)$ \\
\hline$p$-trend & & & $<0.0001$ & & & & 0.30 & & & & 0.46 & & & & 0.71 & & & & 0.21 & \\
\hline$p$-heterogeneity & & & & & & & $<0.0001$ & & & & 0.01 & & & & 0.001 & & & & 0.003 & \\
\hline
\end{tabular}


Table 2 Adjusted odds ratios (OR) and 95\% confidence intervals (CI) for association between breast cancer subtypes associated and reproductive factors (Continued)

\begin{tabular}{|c|c|c|c|c|c|c|c|c|c|c|c|c|c|c|c|c|c|c|c|c|}
\hline \multicolumn{21}{|l|}{ Age at menopause (years) } \\
\hline$<47$ & 2558 & 450 & 1 & Reference & 638 & 136 & 1 & Reference & 333 & 47 & 1 & Reference & 203 & 22 & 1 & Reference & 336 & 61 & 1 & Reference \\
\hline $47-49$ & 2393 & 449 & 1.09 & $(0.94,1.26)$ & 637 & 128 & 0.98 & $(0.74,1.29)$ & 321 & 60 & 1.48 & $(0.96,2.27)$ & 180 & 39 & 2.05 & $(1.14,3.70)$ & 291 & 72 & 1.50 & $(1.01,2.24)$ \\
\hline $50-52$ & 4571 & 870 & 1.11 & $(0.98,1.26)$ & 1162 & 241 & 0.98 & $(0.77,1.24)$ & 644 & 131 & 1.59 & $(1.09,2.32)$ & 324 & 76 & 2.21 & $(1.31,3.75)$ & 611 & 102 & 1.04 & $(0.72,1.49)$ \\
\hline$>52$ & 3051 & 633 & 1.12 & $(0.97,1.28)$ & 840 & 164 & 0.95 & $(0.73,1.24)$ & 415 & 94 & 1.70 & $(1.13,2.55)$ & 202 & 48 & 2.36 & $(1.33,4.21)$ & 395 & 88 & 1.38 & $(0.94,2.01)$ \\
\hline Never (premenopausal) & 1171 & 351 & 2.34 & $(1.93,2.85)$ & 208 & 47 & 1.17 & $(0.76,1.80)$ & 173 & 32 & 1.50 & $(0.86,2.61)$ & 100 & 18 & 1.76 & $(0.81,3.80)$ & 130 & 29 & 1.55 & $(0.88,2.72)$ \\
\hline$p$-trend ${ }^{a}$ & & & 0.12 & & & & 0.72 & & & & 0.01 & & & & 0.004 & & & & 0.38 & \\
\hline$p$-heterogeneity & & & & & & & 0.70 & & & & 0.09 & & & & 0.08 & & & & 0.28 & \\
\hline
\end{tabular}

The category Never was not included in the analysis of $p$-trend. ${ }^{*} p$ for trend and OR mutually adjusted for age $(50-54,55-59,60-64,65-70$ years at screening), body mass index (BMI) $(\leq 22,23-25,26-28,>28$ at screening), education (no education/primary school, high school, bachelor or master's degree), age at menarche $(9-12,13,14,15-18$ years), number of pregnancies (0, 1, 2, 3, $\geq 4)$, menopausal status (premenopausal, perimenopausal, postmenopausal). " $p$ for heterogeneity across subtypes was determined in a case - case model (see "Methods"). ER estrogen receptor, PR progesterone receptor, HER 2 human epidermal growth factor receptor 2 
Table 3 Adjusted odds ratios (OR) and 95\% confidence intervals (Cl) for association between breast cancer subtypes and oral contraceptives and hormone therapy

\begin{tabular}{|c|c|c|c|c|c|c|c|c|c|c|c|c|c|c|c|c|c|c|c|c|}
\hline & \multicolumn{4}{|c|}{$\begin{array}{l}\text { Luminal A-like } \\
\text { ER+PR+ HER2- }\end{array}$} & \multicolumn{4}{|c|}{$\begin{array}{l}\text { Luminal B-like HER2-negative } \\
\text { ER+ PR- HER2- }\end{array}$} & \multicolumn{4}{|c|}{$\begin{array}{l}\text { Luminal B-like HER2-positive } \\
\text { ER+PR+/PR- HER2+ }\end{array}$} & \multicolumn{4}{|c|}{$\begin{array}{l}\text { HER2-positive } \\
\text { ER- PR- HER2+ }\end{array}$} & \multicolumn{4}{|c|}{$\begin{array}{l}\text { Triple-negative } \\
\text { ER- PR- HER2- }\end{array}$} \\
\hline & $\begin{array}{l}\text { Controls } \\
(n)\end{array}$ & $\begin{array}{l}\text { Cases } \\
(n)\end{array}$ & $\mathrm{OR}^{*}$ & $95 \% \mathrm{Cl}$ & $\begin{array}{l}\text { Controls } \\
(n)\end{array}$ & $\begin{array}{l}\text { Cases } \\
(n)\end{array}$ & OR & $95 \% \mathrm{Cl}$ & $\begin{array}{l}\text { Controls } \\
(n)\end{array}$ & $\begin{array}{l}\text { Cases } \\
(n)\end{array}$ & OR & $95 \% \mathrm{Cl}$ & $\begin{array}{l}\text { Controls } \\
(n)\end{array}$ & $\begin{array}{l}\text { Cases } \\
(n)\end{array}$ & OR & $95 \% \mathrm{Cl}$ & $\begin{array}{l}\text { Controls } \\
(n)\end{array}$ & $\begin{array}{l}\text { Cases } \\
(n)\end{array}$ & OR & $95 \% \mathrm{Cl}$ \\
\hline \multicolumn{21}{|c|}{ Age at start of oral contraceptives (years) } \\
\hline $14-18$ & 1476 & 285 & 1 & Reference & 334 & 65 & 1 & Reference & 187 & 45 & 1 & Reference & 128 & 17 & 1 & Reference & 180 & 55 & 1 & Reference \\
\hline $19-20$ & 1462 & 387 & 1.33 & $(1.11,1.59)$ & 367 & 87 & 1.20 & $(0.82,1.75)$ & 230 & 49 & 0.90 & $(0.55,1.45)$ & 133 & 27 & 1.52 & $(0.75,3.10)$ & 206 & 46 & 0.67 & $(0.42,1.07)$ \\
\hline $21-24$ & 1959 & 405 & 1.05 & $(0.87,1.25)$ & 458 & 92 & 1.04 & $(0.71,1.53)$ & 295 & 45 & 0.62 & $(0.37,1.02)$ & 130 & 24 & 1.45 & $(0.69,3.04)$ & 253 & 41 & 0.44 & $(0.27,0.74)$ \\
\hline $25-50$ & 1993 & 397 & 0.99 & $(0.83,1.20)$ & 536 & 102 & 0.97 & $(0.66,1.43)$ & 296 & 58 & 0.80 & $(0.49,1.32)$ & 114 & 27 & 2.23 & $(1.06,4.71)$ & 258 & 58 & 0.63 & $(0.39,1.03)$ \\
\hline Never used & 6715 & 1344 & 1.01 & $(0.86,1.19)$ & 1758 & 363 & 0.99 & $(0.71,1.38)$ & 876 & 180 & 0.82 & $(0.53,1.27)$ & 501 & 115 & 2.05 & $(1.08,3.89)$ & 860 & 165 & 0.54 & $(0.36,0.81)$ \\
\hline$p$-trend ${ }^{\mathrm{a}}$ & & & 0.09 & & & & 0.43 & & & & 0.71 & & & & 0.02 & & & & 0.03 & \\
\hline$p$-heterogeneity & & & & & & & 0.60 & & & & 0.52 & & & & 0.07 & & & & 0.02 & \\
\hline \multicolumn{21}{|c|}{ Duration of oral contraceptives (years) } \\
\hline Never used & 6715 & 1344 & 1 & Reference & 1758 & 363 & 1 & Reference & 876 & 180 & 1 & Reference & 501 & 115 & 1 & Reference & 860 & 165 & 1 & Reference \\
\hline$<2$ & 1839 & 364 & 0.97 & $(0.85,1.11)$ & 482 & 80 & 0.82 & $(0.62,1.08)$ & 275 & 34 & 0.64 & $(0.42,0.97)$ & 151 & 18 & 0.47 & $(0.27,0.81)$ & 263 & 43 & 0.85 & $(0.58,1.26)$ \\
\hline $2-5$ & 1753 & 361 & 1.03 & $(0.90,1.18)$ & 435 & 88 & 1.01 & $(0.77,1.33)$ & 233 & 48 & 0.94 & $(0.64,1.37)$ & 113 & 17 & 0.61 & $(0.34,1.09)$ & 228 & 56 & 1.32 & $(0.92,1.89)$ \\
\hline $6-10$ & 1568 & 332 & 1.06 & $(0.92,1.23)$ & 388 & 80 & 1.03 & $(0.77,1.37)$ & 240 & 56 & 1.23 & $(0.85,1.76)$ & 121 & 35 & 1.07 & $(0.66,1.73)$ & 203 & 47 & 1.20 & $(0.82,1.76)$ \\
\hline$>10$ & 1266 & 298 & 1.11 & $(0.96,1.29)$ & 271 & 62 & 1.12 & $(0.81,1.54)$ & 204 & 45 & 0.95 & $(0.64,1.42)$ & 82 & 19 & 1.02 & $(0.56,1.86)$ & 149 & 38 & 1.25 & $(0.82,1.90)$ \\
\hline$p$-trend* & & & 0.13 & & & & 0.51 & & & & 0.57 & & & & 0.91 & & & & 0.11 & \\
\hline$p$-heterogeneity & & & & & & & 0.61 & & & & 0.10 & & & & 0.003 & & & & 0.56 & \\
\hline \multicolumn{21}{|c|}{ Age at start of intrauterine device (years) } \\
\hline $14-28$ & 612 & 125 & 1 & Reference & 152 & 28 & 1 & Reference & 82 & 14 & 1 & Reference & 55 & 12 & 1 & Reference & 88 & 19 & 1 & Reference \\
\hline $29-35$ & 681 & 143 & 0.97 & $(0.73,1.28)$ & 152 & 39 & 1.31 & $(0.75,2.30)$ & 93 & 10 & 0.76 & $(0.30,1.90)$ & 46 & 7 & 0.74 & $(0.24,2.25)$ & 78 & 25 & 1.42 & $(0.70,2.88)$ \\
\hline $36-42$ & 532 & 147 & 1.33 & $(1.00,1.77)$ & 114 & 21 & 0.83 & $(0.43,1.58)$ & 89 & 19 & 1.34 & $(0.60,3.01)$ & 43 & 7 & 0.75 & $(0.26,2.16)$ & 78 & 12 & 0.70 & $(0.31,1.58)$ \\
\hline $43-50$ & 456 & 121 & 1.16 & $(0.86,1.56)$ & 136 & 25 & 0.86 & $(0.46,1.59)$ & 70 & 11 & 1.06 & $(0.42,2.70)$ & 39 & 7 & 0.94 & $(0.32,2.78)$ & 70 & 14 & 0.94 & $(0.42,2.12)$ \\
\hline Never used & 10000 & 2025 & 0.93 & $(0.75,1.15)$ & 2600 & 529 & 0.94 & $(0.61,1.45)$ & 1368 & 284 & 1.36 & $(0.72,2.56)$ & 729 & 153 & 1.10 & $(0.56,2.16)$ & 1279 & 267 & 0.94 & $(0.55,1.61)$ \\
\hline$p$-trend $d^{a}$ & & & 0.07 & & & & 0.34 & & & & 0.10 & & & & 0.40 & & & & 0.45 & \\
\hline p-heterogeneity ${ }^{\#}$ & & & & & & & 0.22 & & & & 0.12 & & & & 0.20 & & & & 0.38 & \\
\hline \multicolumn{21}{|c|}{ Duration of intrauterine device (years) } \\
\hline Never used & 10000 & 2025 & 1 & Reference & 2600 & 529 & 1 & Reference & 1368 & 284 & 1 & Reference & 729 & 153 & 1 & Reference & 1279 & 267 & 1 & Reference \\
\hline$<2$ & 341 & 64 & 0.91 & $(0.69,1.21)$ & 83 & 16 & 0.86 & $(0.49,1.52)$ & 56 & 13 & 1.23 & $(0.62,2.43)$ & 33 & 5 & 0.84 & $(0.31,2.32)$ & 37 & 7 & 0.90 & $(0.38,2.13)$ \\
\hline $2-5$ & 432 & 104 & 1.17 & $(0.93,1.48)$ & 112 & 21 & 0.95 & $(0.58,1.56)$ & 62 & 11 & 0.96 & $(0.47,1.94)$ & 36 & 7 & 0.81 & $(0.34,1.91)$ & 57 & 21 & 1.79 & $(1.01,3.16)$ \\
\hline $6-10$ & 615 & 140 & 1.16 & $(0.95,1.42)$ & 153 & 40 & 1.37 & $(0.94,2.01)$ & 89 & 15 & 0.86 & $(0.46,1.59)$ & 53 & 8 & 0.62 & $(0.27,1.43)$ & 99 & 20 & 1.23 & $(0.72,2.11)$ \\
\hline$>10$ & 820 & 212 & 1.35 & $(1.14,1.61)$ & 176 & 31 & 0.96 & $(0.63,1.46)$ & 111 & 12 & 0.51 & $(0.27,0.96)$ & 63 & 12 & 0.73 & $(0.37,1.47)$ & 120 & 20 & 0.75 & $(0.44,1.28)$ \\
\hline$p$-trend & & & $<0.0001$ & & & & 0.52 & & & & 0.06 & & & & 0.18 & & & & 0.98 & \\
\hline p-heterogeneity ${ }^{\#}$ & & & & & & & 0.08 & & & & 0.01 & & & & 0.65 & & & & 0.22 & \\
\hline
\end{tabular}


Table 3 Adjusted odds ratios (OR) and 95\% confidence intervals (CI) for association between breast cancer subtypes and oral contraceptives and hormone therapy (Continued) Hormone therapy use

\begin{tabular}{|c|c|c|c|c|c|c|c|c|c|c|c|c|c|c|c|c|c|c|c|c|}
\hline Never & 7411 & 1356 & 1 & Reference & 1762 & 356 & 1 & Reference & 1010 & 195 & 1 & Reference & 573 & 113 & 1 & Reference & 991 & 196 & 1 & Reference \\
\hline Past & 4809 & 1086 & 1.27 & $(1.15,1.40)$ & 1361 & 276 & 0.98 & $(0.81,1.18)$ & 688 & 142 & 1.07 & $(0.81,1.42)$ & 319 & 65 & 0.88 & $(0.60,1.30)$ & 575 & 126 & 1.04 & $(0.79,1.38)$ \\
\hline Estrogen current & 655 & 131 & 1.06 & $(0.86,1.31)$ & 162 & 31 & 0.81 & $(0.53,1.23)$ & 85 & 19 & 1.14 & $(0.65,1.99)$ & 51 & 12 & 1.12 & $(0.54,2.35)$ & 93 & 16 & 0.79 & $(0.43,1.46)$ \\
\hline Estrogen and progesterone current & 345 & 184 & 2.92 & $(2.36,3.62)$ & 89 & 33 & 1.74 & $(1.10,2.74)$ & 48 & 18 & 1.67 & $(0.89,3.14)$ & 32 & 6 & 0.88 & $(0.33,2.30)$ & 49 & 10 & 0.92 & $(0.43,1.98)$ \\
\hline$p$-trend & & & $<0.0001$ & & & & 0.34 & & & & 0.15 & & & & 0.77 & & & & 0.72 & \\
\hline p-heterogeneity ${ }^{\#}$ & & & & & & & 0.05 & & & & 0.34 & & & & 0.03 & & & & 0.01 & \\
\hline \multicolumn{21}{|l|}{ Duration of hormone therapy (years) } \\
\hline Never & 7411 & 1356 & 1 & Reference & 1762 & 356 & 1 & Reference & 1010 & 195 & 1 & Reference & 573 & 113 & 1 & Reference & 991 & 196 & 1 & Reference \\
\hline$<=3$ & 1625 & 306 & 1.09 & $(0.94,1.26)$ & 473 & 96 & 0.99 & $(0.76,1.30)$ & 237 & 43 & 0.90 & $(0.61,1.34)$ & 134 & 20 & 0.66 & $(0.38,1.16)$ & 234 & 41 & 0.77 & $(0.52,1.15)$ \\
\hline $4-8$ & 1130 & 240 & 1.21 & $(1.03,1.43)$ & 289 & 64 & 1.06 & $(0.77,1.46)$ & 155 & 35 & 1.19 & $(0.76,1.87)$ & 76 & 19 & 1.18 & $(0.65,2.13)$ & 119 & 25 & 0.99 & $(0.59,1.67)$ \\
\hline$>8$ & 1756 & 555 & 1.84 & $(1.61,2.11)$ & 485 & 112 & 1.24 & $(0.95,1.61)$ & 253 & 63 & 1.34 & $(0.91,1.98)$ & 108 & 23 & 0.93 & $(0.52,1.65)$ & 222 & 47 & 1.04 & $(0.69,1.56)$ \\
\hline$p$-trend & & & $<0.0001$ & & & & 0.15 & & & & 0.14 & & & & 0.94 & & & & 1.00 & \\
\hline p-heterogeneity ${ }^{\#}$ & & & & & & & 0.001 & & & & 0.48 & & & & 0.11 & & & & 0.02 & \\
\hline \multicolumn{21}{|c|}{ Duration of estrogen and progesterone therapy (years) } \\
\hline Never & 7411 & 1356 & 1 & Reference & 1762 & 356 & 1 & Reference & 1010 & 195 & 1 & Reference & 573 & 113 & 1 & Reference & 991 & 196 & 1 & Reference \\
\hline$<=5$ & 1908 & 412 & 1.25 & $(1.09,1.43)$ & 545 & 114 & 1.02 & $(0.79,1.32)$ & 276 & 45 & 0.79 & $(0.53,1.17)$ & 153 & 30 & 0.92 & $(0.56,1.53)$ & 230 & 54 & 1.19 & $(0.81,1.74)$ \\
\hline$>5$ & 1796 & 561 & 1.80 & $(1.58,2.06)$ & 496 & 116 & 1.18 & $(0.90,1.54)$ & 256 & 71 & 1.47 & $(1.00,2.16)$ & 105 & 29 & 1.04 & $(0.62,1.74)$ & 211 & 52 & 1.34 & $(0.89,2.02)$ \\
\hline$p$-trend & & & $<0.0001$ & & & & 0.31 & & & & 0.27 & & & & 0.98 & & & & 0.13 & \\
\hline p-heterogeneity ${ }^{\#}$ & & & & & & & 0.004 & & & & 0.19 & & & & 0.19 & & & & 0.02 & \\
\hline
\end{tabular}

The category Never was not included in the analysis of $p$-trend. * $p$ for trend and OR mutually adjusted for age $(50-54,55-59,60-64,65-70$ years at screening), body mass index (BMI) $(\leq 22,23-25,26-28,>28$ at screening), education (no education/primary school, high school, bachelor or master's degree), age at menarche $(9-12,13,14,15-18$ years), number of pregnancies $(0,1,2,3, \geq 4)$, menopausal status (premenopausal, perimenopausal, postmenopausal). " $p$ for heterogeneity across subtypes was determined in a case - case model (see "Methods"). ER estrogen receptor, PR progesterone receptor, HER2 human epidermal growth factor receptor 2 
Table 4 Adjusted odds ratios (OR) and 95\% confidence intervals (Cl) for association between breast cancer overall and subtypes of breast cancer, and the duration of estrogen and progestin therapy (EPT) by body mass index (BMI)

\begin{tabular}{|c|c|c|c|c|c|c|c|c|}
\hline \multicolumn{9}{|c|}{ OVERALL breast cancer } \\
\hline & \multicolumn{4}{|l|}{$\mathrm{BMI}<25$} & \multicolumn{4}{|l|}{$\mathrm{BMI} \geq 25$} \\
\hline & Controls $(n)$ & Cases $(n)$ & $\mathrm{OR}^{*}$ & $95 \% \mathrm{Cl}$ & Controls $(n)$ & Cases $(n)$ & OR & $95 \% \mathrm{Cl}$ \\
\hline \multicolumn{9}{|c|}{ Duration of EPT (years) } \\
\hline Never used & 6154 & 1056 & 1 & Reference & 6880 & 1402 & 1 & Reference \\
\hline$<=5$ & 1740 & 316 & 1.20 & $(1.00,1.44)$ & 1812 & 420 & 1.13 & $(0.97,1.32)$ \\
\hline$>5$ & 1623 & 495 & 1.96 & $(1.64,2.34)$ & 1615 & 456 & 1.31 & $(1.12,1.54)$ \\
\hline$p$-trend* & & & $<0.0001$ & & & & $<0.0001$ & \\
\hline$p$ for interaction & & & 0.001 & & & & & \\
\hline
\end{tabular}

Luminal A-like breast cancer

Duration of EPT (years)

\begin{tabular}{|c|c|c|c|c|c|c|c|c|}
\hline Never used & 3424 & 550 & 1 & Reference & 3892 & 789 & 1 & Reference \\
\hline$<=5$ & 959 & 176 & 1.36 & $(1.06,1.74)$ & 987 & 243 & 1.22 & $(0.99,1.50)$ \\
\hline$>5$ & 896 & 293 & 2.29 & $(1.80,2.91)$ & 906 & 270 & 1.38 & $(1.12,1.71)$ \\
\hline$p$-trend & & & $<0.0001$ & & & & 0.002 & \\
\hline
\end{tabular}

$p$ for interaction $\quad 0.001$

Luminal B-like HER2-negative breast cancer

Duration of EPT (years)

$\begin{array}{lllllllll}\text { Never used } & 850 & 173 & 1 & \text { Reference } & 887 & 178 & 1 & \text { Reference } \\ <=5 & 257 & 57 & 1.13 & (0.74,1.71) & 295 & 59 & 0.83 & (0.54,1.29) \\ >5 & 243 & 71 & 1.22 & (0.79,1.88) & 256 & 47 & 0.83 & (0.51,1.35) \\ p \text {-trend } & & & 0.34 & & & & 0.37 & \end{array}$

$p$ for interaction

Luminal B-like HER2-positive breast cancer

Duration of EPT (years)

$\begin{array}{llll}\text { Never used } & 450 & 81 & 1 \\ <=5 & 137 & 16 & 0.85 \\ >5 & 127 & 28 & 1.56 \\ p \text {-trend } & & & 0.44 \\ p \text { for interaction } & & & 0.52\end{array}$

Reference $\quad 544$

$(0.40,1.81) \quad 144$

HER2-positive breast cancer

Duration of EPT (years)

$\begin{array}{ll}\text { Never used } & 269 \\ <=5 & 80 \\ >5 & 49\end{array}$

$p$-trend

$p$ for interaction

Triple-negative breast cancer

Duration of EPT (years)

$\begin{array}{ll}\text { Never used } & 457 \\ <=5 & 123\end{array}$

$(0.67,3.60) \quad 130$

0.44

0.52

$\begin{array}{ll}\text { Reference } & 296 \\ (0.44,2.95) & 77 \\ (0.57,4.05) & 57\end{array}$

Reference

$(0.45,2.04) \quad 110$

522

20
101

34

29

1

Reference

$(0.45,1.49)$

$(0.87,2.78)$

0.24
Reference

$(0.62,3.08)$

$(0.56,2.93)$ 
Table 4 Adjusted odds ratios (OR) and 95\% confidence intervals (Cl) for association between breast cancer overall and subtypes of breast cancer, and the duration of estrogen and progestin therapy (EPT) by body mass index (BMI) (Continued)

$\begin{array}{lllllllll}>5 & 113 & 25 & 1.27 & (0.56,2.86) & 100 & 27 & 1.34 & (0.71,2.54) \\ p \text {-trend } & & & 0.63 & & & 0.25 & \\ p \text { for interaction } & & & 0.09 & & & & & \end{array}$

* $p$ for trend and ORs mutually adjusted for age $(50-54,55-59,60-64,65-70$ years at screening), BMl ( $\leq 22,23-25,26-28,>28$ at screening), age at menarche $(9-12,13,14,15-18)$, education (no education/primary school, high school, bachelor's or master's degree), number of pregnancies (0, 1, 2, 3, $\geq 4)$, menopausal status (premenopausal, perimenopausal, postmenopausal). HER2 human epidermal growth factor receptor 2

largest single studies of breast cancer subtypes. The number of previous pregnancies was associated with a decreased risk, and late age at first birth was associated with an increased risk of luminal-like subtypes. Although not statistically significant, number of pregnancies and age at first birth were also associated with HER2-positive and triple-negative breast cancer. There were larger differences between subtypes with the use of exogenous hormones. Duration of oral contraceptive use and IUDs were weakly associated with luminal A-like breast cancer, while current EPT was associated with an almost threefold increased risk of luminal A-like breast cancer, but was not associated with either HER2-positive or triple-negative cancer.

There have been different methods of classifying breast cancer subtypes. In our study we used the classification from the St. Gallen meeting in 2013 [46] where we included only ER+ PR+ HER2- as luminal A, while ER+ PR- HER2- was classified as one of the luminal $B$ definitions, while the second luminal B subtype was the one that is more commonly referred to as luminal $\mathrm{B}$, i.e. $\mathrm{ER}+$ and/or $\mathrm{PR}+$ HER2+. In contrast, in the systematic review of 38 studies [47] they used a wider definition of luminal A (ER+ and/or PR+ and HER2-), and only one luminal $\mathrm{B}$ subtype (ER+ and/or $\mathrm{PR}+\mathrm{HER} 2+)$. Some studies have also added on Ki67 to the definition of luminal B $[40,48,49]$, which may give a more precise definition of luminal B, although Ki67 is notoriously difficult to assess [55]. Although our luminal A results were much in line with previous studies, there were some slight differences between our study and previous studies of luminal B, as discussed below.

In the current study we also examined triple-negative breast cancer (ER- PR- HER2-). Some studies have added additional markers, to better define the subset that is basal-like, by HER $1+$ and/or cytokeratin $5 / 6+[9$, $39,40,49,56]$. As discussed subsequently, this may explain some discrepancies between the results of different studies.

\section{Reproductive factors}

Our observation of an inverse association between parity and luminal A-like breast cancer is consistent with the vast majority of studies as summarized in the recent systematic review of 38 studies [47] and the more recent studies that were not included in the review [7, 8, 10,48-51]. The results for luminal $B$ breast cancer have been less clear, with studies finding a protective or increased effect, or no effect of parity [47-50]. In the current study, parity was associated with a decreased risk of both luminal Blike subtypes. This is consistent with four out of six studies included in the systematic review [47] and three of the more recent studies [48-50]. Two of these recent studies had information on Ki-67 [48, 49]. When we added grade to the luminal A-like and luminal B-like subtypes, the parity results remained largely the same. These results suggest that there is a protective effect against luminal B, and the effect seems apparent regardless of the markers used to define the luminal $B$ subtype.

Although not statistically significant, we found that parity was associated with a decreased risk of HER2positive breast cancer. This is consistent with the findings in the Nurses' Health Study [49], but inconsistent with a case-control study from the Breast Cancer Family Registry [50] and a Korean cohort study [48], which reported that parity was associated with an increased risk of the HER2-positive subtype. One of these latter studies included very few women with HER2-positive breast cancer and both studies included younger women than the current study.

However, although not statistically significant, we found that parity was associated with a decreased risk of triple-negative breast cancer.

This is inconsistent with several other studies in which parity was associated with no risk [48], or was associated with increased risk of triple-negative breast cancer [7, 47, 50]. One of the studies was in African American women who are less likely to breastfeed compared to Caucasian women [7]. Several studies have used CK5/6 and epithelial growth factor receptor (EGFR) in addition to ER, PR and HER2neu to define a basal-like subtype $[9,39,40,49,56]$, and this may be one reason for the inconsistency between our study and these other studies. We included 386 women with triple-negative breast cancer in our study, but few of these had never been pregnant $(n=34)$ or had four or more children $(n=36)$. More 
notably, our study consisted of older women (aged 50-69 years). Although we did not have information on time since the last pregnancy, we can only speculate that few women in our study had had a recent pregnancy, a factor associated with increased risk of triple-negative cancer [57]. Perhaps this explains why we did not find an increased risk of triple-negative cancer with multi-parity.

We found that age at first birth (26 years or older) was associated with an increased risk of luminal-like breast cancer. This is consistent with a systematic review and [47] and two recent studies [8, 49]. However, age at first birth was only statistically significantly associated with luminal A cancer, whereas in the current study age at first birth was significantly associated with luminal A-like and luminal B-like HER2-negative subtypes. One reason for this difference might be that our definition of luminal B-like HER2-negative cancer was rather similar to the definition of the luminal A-like subtype used in the other studies. In the current study, a non-statistically significant positive association was observed between late age at first birth (31 years or older), and both HER2-positive and triple-negative breast cancer. This is consistent with the Nurses' Health Study [49], and inconsistent with the case-control study from Korea [8]. In the latter study, the majority of women were premenopausal, whereas the current study included mainly postmenopausal women.

We found no protective effect of breastfeeding on breast cancer occurrence overall or for any subtype. This is inconsistent with the systematic review that reported that breastfeeding is associated with decreased risk of luminal A-like, luminal B-like and triple-negative subtypes [47]. This may be because our study only included women above 50 years of age, and is consistent with the suggestion that the protective effect of breastfeeding is relatively timelimited, and may be seen predominantly in younger women [58-60]. Several of the more recent studies found a significant protective effect against basal-like breast cancer [7, 49-51]. These studies included both triple-negative breast cancer as in our study and basal-like breast cancer with more markers (CK 5/6 and EGFR) [49].

\section{Oral contraceptives, intrauterine devices, and menopausal} hormone therapy use

For long duration of oral contraceptive use ( $>10$ years), we observed a slight increase in risk of all the subtypes except for the luminal B-like HER2-positive and HER2positive subtypes. Our positive association between duration of oral contraceptive use and triple-negative breast cancer is consistent with the systematic review [47]. However, two of the three studies in the review reported a decreased risk between oral contraceptive use and the luminal A subtype $[9,34]$. These latter studies were smaller and included younger women (20-74 and $<56$ years old) than the current study. There are few data on IUD use. Two studies reported that IUDs were not associated with an increased risk of breast cancer [61, 62], whereas other studies report that IUDs were associated with an increased breast cancer risk [63, 64]. We observed a significant positive trend for association between duration of IUD use and breast cancer overall and luminal A-like breast cancer. However, the increased risk of luminal A-like breast cancer was only statistically significant in women using IUDs for more than 10 years. This is consistent with two Finnish studies of levonorgestrel-releasing IUDs and breast cancer [63, 64], which reported that levonorgestrel-releasing IUDs were associated with an increased risk of breast cancer. One hypothesis is that levonorgestrel-releasing IUDs have substantial progestogenic and androgenic effects [63, 64], which could contribute to this increased risk with IUD use.

We observed a large increased risk of luminal A-like and a moderate increased risk of luminal B-like breast cancer with use of menopausal hormone therapy, albeit only significantly so for the luminal A-like subtype. This is consistent with a systematic review [47] and the cohort study from the Nurses' Health Study that investigated only one luminal B subtype, and used Ki-67 to differentiate between luminal A-like and luminal B-like subtypes [49]. There was some evidence that menopausal hormone therapy was associated with a slightly decreased risk of triple-negative breast cancer when we compared current menopausal hormone therapy users to never users. This is consistent with one of the studies in the systematic review, which was a case-control study from Washington state [35]. The latter study used the same definition of triple-negative breast cancer as the current study. Inconsistent with our result, studies from the Women's Health Initiative [65] and the Nurses' Health Study $[40,49]$ reported an association between menopausal hormone therapy and increased risk of triple-negative breast cancer. In these latter studies, they used more biomarkers (CK 5/6 and EGFR) to define the basal-like subtype and included younger women than the current study. When we looked at the duration of EPT, compared to never users, women who had used EPT for more than 5 years were at an increased risk of HER2-positive and triple-negative breast cancer. This is consistent with the analysis from the Nurses' Health Study [49].

We hypothesized that EPT would have a stronger effect on thin women than on heavier women, and our results suggested modification of the effect of the duration of EPT when we analyzed leaner women (BMI 
$<25)$ and heavier women (BMI $>25)$ with the luminallike subtypes of breast cancer. This is consistent with a previous population-based case-control study of women aged $55-72$ years [66].

\section{Mechanisms and suggested subtype differences}

Pregnancies have been reported to ultimately reduce plasma estrogen (estrone, estradiol and estriol) [67] and follicular-phase progesterone [68], and increase sex hormone-binding globulin [67]. Our findings suggest that both parity and combined menopausal hormone therapy may be predominantly associated with luminallike breast tumors, with the association being strongest for the luminal A-like subtype. The effect of EPT was stronger when we added grade to the definition of luminal A cancer. We observed little effect of breastfeeding overall, and no clear subtype differences. It is possible that the effect of breastfeeding is nonhormonal, and includes changes in immune responses and apoptosis $[69,70]$.

Our results, together with those from other large studies, further suggest that the associations between these hormonal-related factors (parity, age at first birth, oral contraceptive use and menopausal hormone therapy use) and risk of the luminal B-like subtypes are similar to the associations between these factors and risk of the luminal A-like subtype. However, although the association with luminal A was the strongest, it is clear that EPT also increases the risk of breast cancer with bad prognosis [71].

We observed some intriguing associations with HER2positive breast cancer. The effect of pregnancies and age at first birth were similar for the HER2-positive subtype and luminal cancer. Also, age at menopause was strongly associated with increased risk only of the HER2-positive subtype. Further, we observed a positive trend of association between age at menopause and the luminal B-like HER2-positive subtype. These results might imply that there are hormonal mechanisms involved in the expression of the HER2 protein. On the other hand, exogenous hormone use (EPT) was not associated with this subtype, suggesting that perhaps only some hormonal mechanisms play a role in HER2-positive subtypes. The contrasting results between the luminal-like subtypes and triple-negative breast cancer are also consistent with previous literature. Hormonal factors have a stronger effect on ER+ PR+ tumors, which suggests that the etiology of triple-negative cancer is different from that of the luminal subtypes. Specifically, this suggests that triple-negative tumors may not be as easily prevented hormonally.

In summary, the strongest discrepancy across subtypes was for the use of combined hormone therapy, where the effect was clearly much stronger for luminal A-like than for other cancers. Otherwise, the associations with hormonal risk factors were stronger for luminal $\mathrm{A}$ and B-like subtypes than for HER2-positive and triplenegative subtypes. Our results suggest that reproductive factors may to some extent be associated with HER2positive tumors, but that triple-negative tumors have a different etiology.

\section{Strengths and limitations}

Strengths of this study include its population-based design, the large size, being the largest single study of breast cancer subtypes conducted so far, and the availability of prospectively collected detailed information on many risk factors for breast cancer.

Although the study is the largest to date, there was still limited power for analysis of the rare breast cancer subtypes. Another weakness was the lack of molecular expression data. This may have obscured differences between the subtypes. Another possible weakness was that a subset of women were diagnosed within a month of completing the questionnaire $(n=$ 1813), and could have been symptomatic when they completed their questionnaire. However, exclusion of women who were diagnosed within a month of completing the questionnaire did not affect the results (results not shown).

Women who attend screening might be more health-conscious and have a healthier lifestyle than women who do not attend. This could have contributed to obliteration of protective effects of "healthy" habits, such as an effect of breastfeeding overall. At the same time, women who attend screening are more likely to have breast cancer detected. Thus, the picture becomes complicated with these potential biases, and it is not clear how this would explain the results of this paper. The associations between wellestablished risk factors and overall risk of breast cancer were largely as expected. Furthermore, it is unlikely that any such bias would have differentially affected the results for different subtypes.

\section{Conclusions}

Reproductive factors and menopausal hormone therapy use were more strongly associated with luminallike breast cancer, but reproductive factors were also associated with HER2-positive and triple-negative breast cancer. The differences between subtypes were greatest for menopausal hormone use. Our results add to the literature showing that there are etiologic differences between luminal breast cancer subtypes and basal-like or triple-negative breast cancer subtypes, but suggest that the differences may be limited. 


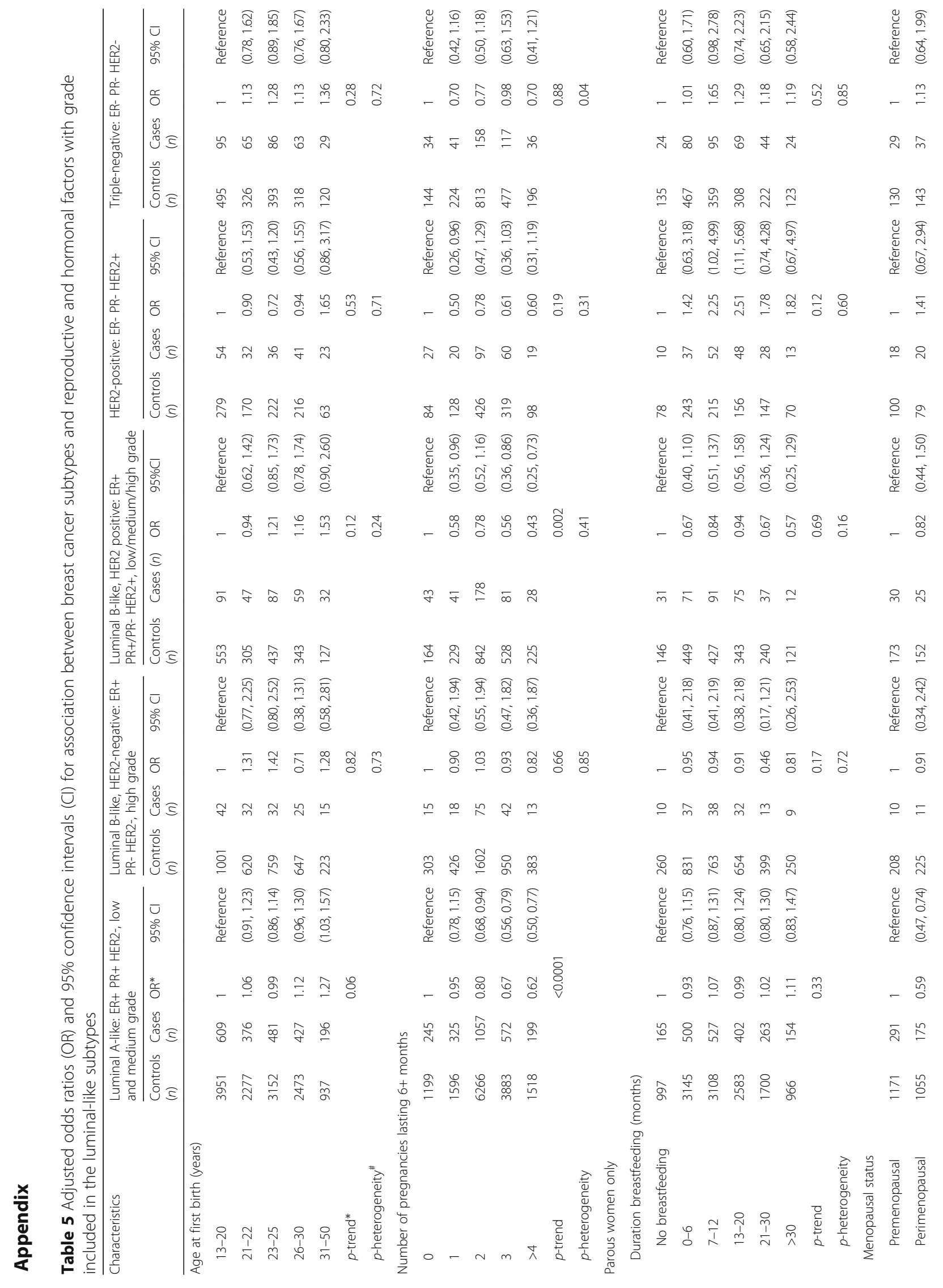




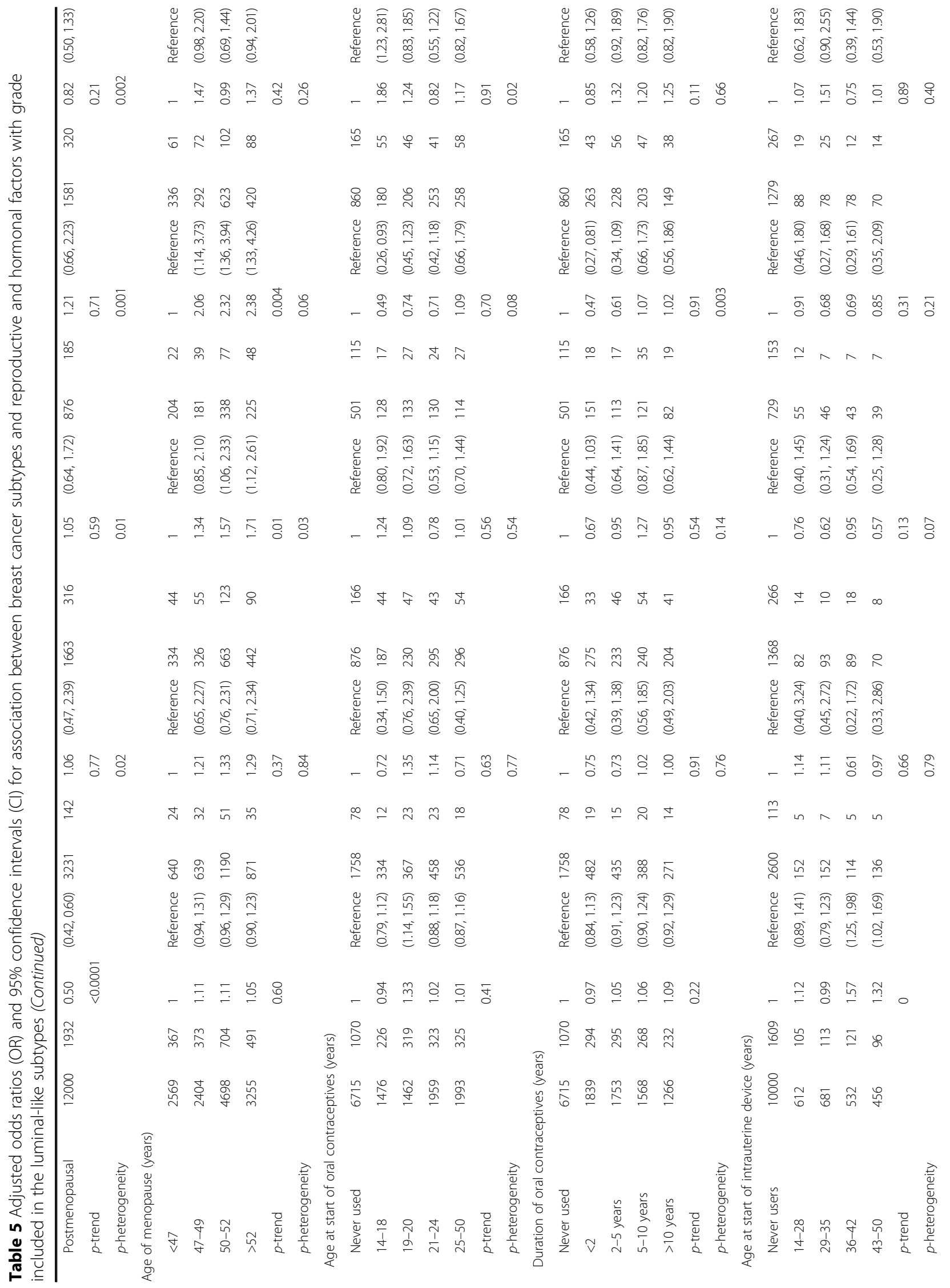




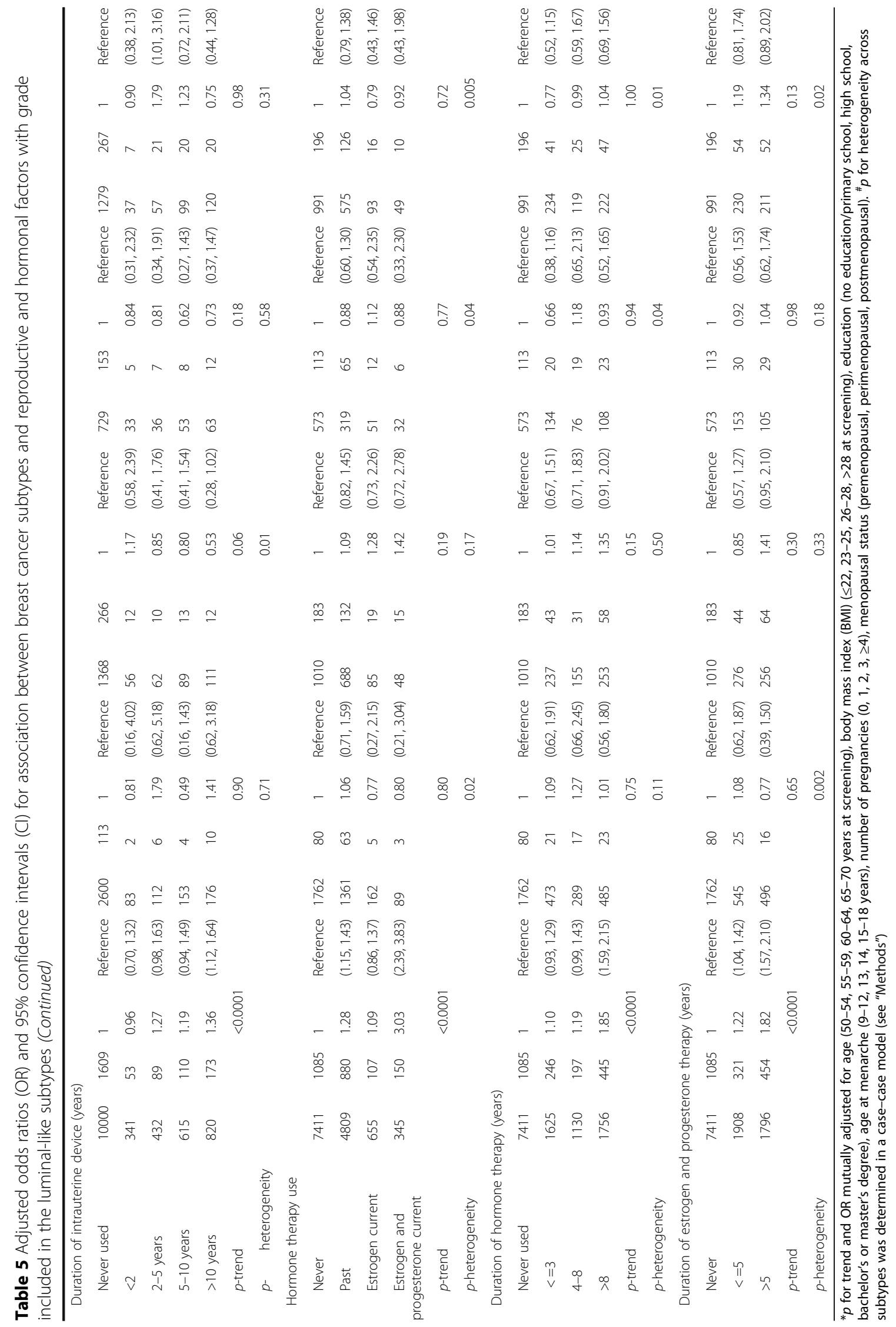




\section{Abbreviations}

BMl: body mass index; $\mathrm{Cl}$ : confidence interval; $\mathrm{CISH}$ : chromogenic in situ hybridization; CRN: The Cancer Registry of Norway; EPT: estrogen and progesterone therapy; ER: estrogen receptor; FISH: fluorescence in situ hybridization; HER2: human epidermal growth factor receptor 2; IHC: immunohistochemical analysis; PR: progesterone receptor; SISH: silver in situ hybridization

\section{Acknowledgements}

Not applicable.

\section{Funding}

The study was funded by the Norwegian Cancer Society, which had no role in the design, collection of data, analysis or interpretation of the study.

\section{Availability of data and material}

The data supporting the findings of this study are available at the Cancer Registry of Norway for investigators with the appropriate Norwegian approvals, but restrictions apply. However, data can be made available from the authors upon reasonable request, and with the permission of the Norwegian Regional Committee for Medical and Health Research Ethics.

\section{Authors' contributions}

MED and LV cleaned the data and ran the statistical analyses and MED drafted the manuscript. LV, ST, SH, and IdSS contributed to the design of the study and interpretation of data and helped revised the manuscript. GU conceived the study and supervised the design, statistical analysis, and manuscript preparation. All authors have read and approved the final manuscript.

\section{Competing interests}

The authors declare that they have no competing interests.

\section{Consent for publication}

Not applicable.

\section{Ethics approval and consent to participate}

Participants were informed that submission of a completed questionnaire indicated that they gave their consent to participate in studies of breast cancer. The study was approved by the Regional Committee for Medical and Health Research Ethics in the South-East Health Region of Norway (2014/1167)

\section{Author details}

${ }^{1}$ Cancer Registry of Norway, Oslo, Norway. ${ }^{2}$ Department of Non-Communicable Disease Epidemiology, London School of Hygiene and Tropical Medicine, London, UK. ${ }^{3}$ University of Oslo, Oslo, Norway. ${ }^{4}$ University of Southern California, Los Angeles, CA, USA.

\section{Received: 12 August 2016 Accepted: 22 December 2016}

\section{Published online: 23 January 2017}

\section{References}

1. Collaborative Group on Hormonal Factors in Breast Cancer. Breast cancer and breastfeeding: collaborative reanalysis of individual data from 47 epidemiological studies in 30 countries, including 50302 women with breast cancer and 96973 women without the disease. Lancet. 2002; 360(9328):187-95.

2. Kelsey JL, Gammon MD, John EM. Reproductive factors and breast cancer. Epidemiol Rev. 1993;15(1):36-47.

3. Kwan ML, Bernard PS, Kroenke CH, Factor RE, Habel LA, Weltzien EK, Castillo A, Gunderson EP, Maxfield KS, Stijleman IJ et al. Breastfeeding, PAM50 tumor subtype, and breast cancer prognosis and survival. J Natl Cancer Inst. 2015; 107(7):djv087.

4. Gierisch JM, Coeytaux RR, Urrutia RP, Havrilesky $\sqcup$, Moorman PG, Lowery WJ, Dinan M, McBroom AJ, Hasselblad V, Sanders GD, et al. Oral contraceptive use and risk of breast, cervical, colorectal, and endometrial cancers: a systematic review. Cancer Epidem Biomar. 2013;22(11):1931-43.

5. Beral V, Reeves G. Childbearing, oral contraceptive use, and breast cancer. Lancet. 1993;341(8852):1102.

6. Evans G, Sutton EL. Oral Contraception. Med Clin N Am. 2015;99(3):479-503.
7. Ambrosone CB, Zirpoli G, Ruszczyk M, Shankar J, Hong CC, Mcllwain D, Roberts M, Yao S, McCann SE, Ciupak G, et al. Parity and breastfeeding among African-American women: differential effects on breast cancer risk by estrogen receptor status in the Women's Circle of Health Study. Cancer Causes Control. 2014;25(2):259-65.

8. Park B, Choi JY, Sung HK, Ahn C, Hwang Y, Jang J, Lee J, Kim H, Shin HR, Park $\mathrm{S}$, et al. Attribution to heterogeneous risk factors for breast cancer subtypes based on hormone receptor and human epidermal growth factor 2 receptor expression in Korea. Medicine. 2016;95(14), e3063.

9. Razzaghi H, Troester MA, Gierach GL, Olshan AF, Yankaskas BC, Millikan RC. Association between mammographic density and basal-like and luminal A breast cancer subtypes. Breast Cancer Res. 2013;15(5):R76.

10. Cui Y, Deming-Halverson SL, Shrubsole MJ, Beeghly-Fadiel A, Fair AM, Sanderson M, Shu XO, Kelley MC, Zheng W. Associations of hormone-related factors with breast cancer risk according to hormone receptor status among white and African American women. Clin Breast Cancer. 2014;14(6):417-25.

11. Hirko KA, Chen WY, Willett WC, Rosner BA, Hankinson SE, Beck AH, Tamimi $\mathrm{RM}$, Eliassen AH. Alcohol consumption and risk of breast cancer by molecular subtype: prospective analysis of the nurses' health study after 26 years of follow-up. Int J Cancer. 2016;138(5):1094-101.

12. Fagherazzi G, Vilier A, Boutron-Ruault MC, Mesrine S, Clavel-Chapelon F. Alcohol consumption and breast cancer risk subtypes in the E3N-EPIC cohort. Eur J Cancer Prev. 2015;24(3):209-14.

13. Wong AW, Dunlap SM, Holcomb VB, Nunez NP. Alcohol promotes mammary tumor development via the estrogen pathway in estrogen receptor alphanegative HER2/neu mice. Alcohol Clin Exp Res. 2012;36(4):577-87.

14. Ke Z, Lin H, Fan Z, Cai TQ, Kaplan RA, Ma C, Bower KA, Shi X, Luo J. MMP-2 mediates ethanol-induced invasion of mammary epithelial cells overexpressing ErbB2. Int J Cancer. 2006;119(1):8-16.

15. Chlebowski RT, Anderson GL, Gass M, Lane DS, Aragaki AK, Kuller LH, Manson JE, Stefanick ML, Ockene J, Sarto GE, et al. Estrogen plus progestin and breast cancer incidence and mortality in postmenopausal women. JAMA. 2010;304(15):1684-92.

16. Dumitrescu RG, Shields PG. The etiology of alcohol-induced breast cancer. Alcohol. 2005;35(3):213-25.

17. Parker JS, Mullins M, Cheang MC, Leung S, Voduc D, Vickery T, Davies S, Fauron C, He X, Hu Z, et al. Supervised risk predictor of breast cancer based on intrinsic subtypes. J Clin Oncol. 2009;27(8):1160-7.

18. Perou CM, Sorlie T, Eisen MB, van de Rijn M, Jeffrey SS, Rees CA, Pollack JR, Ross DT, Johnsen $\mathrm{H}$, Akslen LA, et al. Molecular portraits of human breast tumours. Nature. 2000;406(6797):747-52.

19. Sorlie T, Perou CM, Tibshirani R, Aas T, Geisler S, Johnsen H, Hastie T, Eisen $M B$, van de Rijn M, Jeffrey SS, et al. Gene expression patterns of breast carcinomas distinguish tumor subclasses with clinical implications. Proc Natl Acad Sci U S A. 2001;98(19):10869-74

20. Sorlie T, Tibshirani R, Parker J, Hastie T, Marron JS, Nobel A, Deng S, Johnsen $H$, Pesich R, Geisler S, et al. Repeated observation of breast tumor subtypes in independent gene expression data sets. Proc Natl Acad Sci U S A. 2003; 100(14):8418-23.

21. Sorlie T, Wang Y, Xiao C, Johnsen H, Naume B, Samaha RR, Borresen-Dale AL. Distinct molecular mechanisms underlying clinically relevant subtypes of breast cancer: gene expression analyses across three different platforms. BMC Genomics. 2006;7:127.

22. Van 't Veer $L$, De Jong $D$. The microarray way to tailored cancer treatment. Nat Med. 2002;8(1):13-4.

23. van't Veer $L$, Dai HY, van de Vijver MJ, He YDD, Hart AAM, Mao M, Peterse $\mathrm{HL}$, van der Kooy K, Marton MJ, Witteveen AT, et al. Gene expression profiling predicts clinical outcome of breast cancer. Nature. 2002;415(6871): 530-6.

24. Britton JA, Gammon MD, Schoenberg JB, Stanford JL, Coates RJ, Swanson CA, Potischman N, Malone KE, Brogan DJ, Daling JR, et al. Risk of breast cancer classified by joint estrogen receptor and progesterone receptor status among women 20-44 years of age. Am J Epidemiol. 2002;156(6):507-16.

25. Ma HY, Bernstein L, Pike MC, Ursin G. Reproductive factors and breast cancer risk according to joint estrogen and progesterone receptor status: a meta-analysis of epidemiological studies. Breast Cancer Res. 2006;8(4):R43.

26. Warner ET, Tamimi RM, Boggs DA, Rosner B, Rosenberg L, Colditz GA, Palmer JR. Estrogen receptor positive tumors: do reproductive factors explain differences in incidence between black and white women? Cancer Causes Control. 2013;24(4):731-9. 
27. Ritte R, Lukanova A, Berrino F, Dossus L, Tjonneland A, Olsen A, Overvad TF, Overvad K, Clavel-Chapelon F, Fournier A, et al. Adiposity, hormone replacement therapy use and breast cancer risk by age and hormone receptor status: a large prospective cohort study. Breast Cancer Res. 2012; 14(3):R76.

28. Bao PP, Shu XO, Gao YT, Zheng Y, Cai H, Deming SL, Ruan ZX, Su Y, Gu K, Lu W, et al. Association of hormone-related characteristics and breast cancer risk by estrogen receptor/progesterone receptor status in the Shanghai breast cancer study. Am J Epidemiol. 2011;174(6):661-71.

29. Ma H, Henderson KD, Sullivan-Halley J, Duan L, Marshall SF, Ursin G, Horn-Ross PL, Largent J, Deapen DM, Lacey Jr JV, et al. Pregnancyrelated factors and the risk of breast carcinoma in situ and invasive breast cancer among postmenopausal women in the California Teachers Study cohort. Breast Cancer Res. 2010;12(3):R35.

30. Setiawan WW, Monroe KR, Wilkens LR, Kolonel LN, Pike MC, Henderson BE. Breast cancer risk factors defined by estrogen and progesterone receptor status: the multiethnic cohort study. Am J Epidemiol. 2009;169(10):1251-9.

31. Li Cl, Malone KE, Porter PL, Weiss NS, Tang MTC, Cushing-Haugen KL, Daling $J R$. Relationship between long durations and different regimens of hormone therapy and risk of breast cancer. JAMA. 2003;289(24):3254-63.

32. Rosato V, Bosetti C, Negri E, Talamini R, Dal Maso L, Malvezzi M, Falcini F, Montella M, La Vecchia C. Reproductive and hormonal factors, family history, and breast cancer according to the hormonal receptor status. Eur J Cancer Prev. 2014;23(5):412-7.

33. Dolle JM, Daling JR, White E, Brinton LA, Doody DR, Porter PL, Malone KE. Risk factors for triple-negative breast cancer in women under the age of 45 years. Cancer Epidemiol Biomarkers Prev. 2009;18(4):1157-66.

34. Gaudet MM, Press MF, Haile RW, Lynch CF, Glaser SL, Schildkraut J, Gammon MD, Douglas Thompson W, Bernstein JL. Risk factors by molecular subtypes of breast cancer across a population-based study of women 56 years or younger. Breast Cancer Res Treat. 2011;130(2):587-97.

35. Phipps Al, Malone KE, Porter PL, Daling JR, Li Cl. Reproductive and hormonal risk factors for postmenopausal luminal, HER-2-overexpressing, and triplenegative breast cancer. Cancer. 2008;113(7):1521-6.

36. Xing P, Li J, Jin F. A case-control study of reproductive factors associated with subtypes of breast cancer in Northeast China. Med Oncol. 2010;27(3): 926-31.

37. Martinez ME, Wertheim BC, Natarajan L, Schwab R, Bondy M, DaneriNavarro A, Meza-Montenegro MM, Gutierrez-Millan LE, Brewster A, Komenaka IK, et al. Reproductive factors, heterogeneity, and breast tumor subtypes in women of Mexican descent. Cancer Epidemiol Biomarkers Prev. 2013;22(10):1853-61.

38. Li Cl, Beaber EF, Tang MT, Porter PL, Daling JR, Malone KE. Reproductive factors and risk of estrogen receptor positive, triple-negative, and HER2-neu overexpressing breast cancer among women 20-44 years of age. Breast Cancer Res Treat. 2013;137(2):579-87.

39. Millikan RC, Newman B, Tse CK, Moorman PG, Conway K, Dressler LG, Smith LV, Labbok MH, Geradts J, Bensen JT, et al. Epidemiology of basal-like breast cancer. Breast Cancer Res Treat. 2008;109(1):123-39.

40. Tamimi RM, Colditz GA, Hazra A, Baer HJ, Hankinson SE, Rosner B, Marotti J, Connolly JL, Schnitt SJ, Collins LC. Traditional breast cancer risk factors in relation to molecular subtypes of breast cancer. Breast Cancer Res Treat. 2012;131(1):159-67.

41. Horn J, Opdahl S, Engstrom MJ, Romundstad PR, Tretli S, Haugen OA, Bofin $A M$, Vatten $L J$, Asvold BO. Reproductive history and the risk of molecular breast cancer subtypes in a prospective study of Norwegian women. Cancer Causes Control. 2014;25(7):881-9.

42. Ma HY, Wang YP, Sullivan-Halley J, Weiss L, Marchbanks PA, Spirtas R, Ursin G, Burkman RT, Simon MS, Malone KE, et al. Use of four biomarkers to evaluate the risk of breast cancer subtypes in the Women's Contraceptive and Reproductive Experiences Study. Cancer Res. 2010;70(2):575-87.

43. Yang XR, Pfeiffer RM, Garcia-Closas M, Rimm DL, Lissowska J, Brinton LA, Peplonska B, Hewitt SM, Cartun RW, Mandich D, et al. Hormonal markers in breast cancer: coexpression, relationship with pathologic characteristics, and risk factor associations in a population-based study. Cancer Res. 2007;67(21): 10608-17.

44. Yang XR, Chang-Claude J, Goode EL, Couch FJ, Nevanlinna H, Milne RL, Gaudet M, Schmidt MK, Broeks A, Cox A, et al. Associations of breast cancer risk factors with tumor subtypes: a pooled analysis from the Breast Cancer Association Consortium studies. J Natl Cancer Inst. 2011;103(3):250-63.
45. Perou CM, Jeffrey SS, van de Rijn M, Rees CA, Eisen MB, Ross DT, Pergamenschikov A, Williams CF, Zhu SX, Lee JC, et al. Distinctive gene expression patterns in human mammary epithelial cells and breast cancers. Proc Natl Acad Sci U S A. 1999;96(16):9212-7.

46. Goldhirsch A, Winer EP, Coates AS, Gelber RD, Piccart-Gebhart M, Thurlimann B, Senn HJ, Panel M. Personalizing the treatment of women with early breast cancer: highlights of the St Gallen International Expert Consensus on the Primary Therapy of Early Breast Cancer 2013. Ann Oncol. 2013;24(9):2206-23.

47. Barnard ME, Boeke CE, Tamimi RM. Established breast cancer risk factors and risk of intrinsic tumor subtypes. Biochim Biophys Acta. 2015;1856(1):73-85.

48. Lee SK, Kim SW, Han SA, Kil WH, Lee JE, Nam SJ. The protective effect of parity in hormone receptor-positive, Ki-67 expressing breast cancer. World J Surg. 2014;38(5):1065-9.

49. Sisti JS, Collins LC, Beck AH, Tamimi RM, Rosner BA, Eliassen AH. Reproductive risk factors in relation to molecular subtypes of breast cancer: results from the nurses' health studies. Int J Cancer. 2016; 138(10):2346-56.

50. Work ME, John EM, Andrulis IL, Knight JA, Liao Y, Mulligan AM, Southey MC, Giles GG, Dite GS, Apicella C, et al. Reproductive risk factors and oestrogen/ progesterone receptor-negative breast cancer in the Breast Cancer Family Registry. Br J Cancer. 2014;110(5):1367-77.

51. Islami F, Liu Y, Jemal A, Zhou J, Weiderpass E, Colditz G, Boffetta P, Weiss M. Breastfeeding and breast cancer risk by receptor status-a systematic review and meta-analysis. Ann Oncol. 2015;26(12):2398-407.

52. Kwan ML, Kroenke CH, Sweeney C, Bernard PS, Weltzien EK, Castillo A, Factor RE, Maxfield KS, Stijleman IJ, Kushi LH, et al. Association of high obesity with PAM50 breast cancer intrinsic subtypes and gene expression. BMC Cancer. 2015;15:278.

53. Hofvind S, Geller B, Vacek PM, Thoresen S, Skaane P. Using the European guidelines to evaluate the Norwegian Breast Cancer Screening Program. Eur J Epidemiol. 2007;22(7):447-55.

54. Larsen IK, Smastuen M, Johannesen TB, Langmark F, Parkin DM, Bray F, Moller B. Data quality at the Cancer Registry of Norway: an overview of comparability, completeness, validity and timeliness. Eur J Cancer. 2009; 45(7):1218-31.

55. Polley MY, Leung SC, McShane LM, Gao D, Hugh JC, Mastropasqua MG, Viale G, Zabaglo LA, Penault-Llorca F, Bartlett JM, et al. An international Ki67 reproducibility study. J Natl Cancer Inst. 2013;105(24):1897-906.

56. Yang XR, Sherman ME, Rimm DL, Lissowska J, Brinton LA, Peplonska B, Hewitt SM, Anderson WF, Szeszenia-Dabrowska N, Bardin-Mikolajczak A, et al. Differences in risk factors for breast cancer molecular subtypes in a population-based study. Cancer Epidemiol Biomarkers Prev. 2007;16(3): 439-43.

57. Amant F, von Minckwitz G, Han SN, Bontenbal M, Ring AE, Giermek J, Wildiers H, Fehm T, Linn SC, Schlehe B, et al. Prognosis of women with primary breast cancer diagnosed during pregnancy: results from an international collaborative study. J Clin Oncol. 2013;31(20):2532-9.

58. Byers T, Graham S, Rzepka T, Marshall J. Lactation and breast cancer. Evidence for a negative association in premenopausal women. Am J Epidemiol. 1985;121(5):664-74.

59. McTiernan A, Thomas DB. Evidence for a protective effect of lactation on risk of breast cancer in young women. Results from a case-control study. Am J Epidemiol. 1986;124(3):353-8.

60. Brignone G, Cusimano R, Dardanoni G, Gugliuzza M, Lanzarone F, Scibilia V, Dardanoni L. A case-control study on breast cancer risk factors in a southern European population. Int J Epidemiol. 1987;16(3): 356-61.

61. Dinger J, Bardenheuer K, Minh TD. Levonorgestrel-releasing and copper intrauterine devices and the risk of breast cancer. Contraception. 2011;83(3): 211-7.

62. Backman T, Rauramo I, Jaakkola K, Inki P, Vaahtera K, Launonen A, Koskenvuo M. Use of the levonorgestrel-releasing intrauterine system and breast cancer. Obstet Gynecol. 2005;106(4):813-7.

63. Lyytinen HK, Dyba T, Ylikorkala O, Pukkala El. A case-control study on hormone therapy as a risk factor for breast cancer in Finland: intrauterine system carries a risk as well. Int J Cancer. 2010;126(2):483-9.

64. Soini T, Hurskainen R, Grenman S, Maenpaa J, Paavonen J, Joensuu H, Pukkala E. Levonorgestrel-releasing intrauterine system and the risk of breast cancer: A nationwide cohort study. Acta Oncol. 2016;55(2): 188-92. 
65. Islam T, Matsuo K, Ito H, Hosono S, Watanabe M, Iwata H, Tajima K, Tanaka H. Reproductive and hormonal risk factors for luminal, HER2-overexpressing, and triple-negative breast cancer in Japanese women. Ann Oncol. 2012; 23(9):2435-41.

66. Ursin G, Tseng CC, Paganini-Hill A, Enger S, Wan PC, Formenti S, Pike MC, Ross RK. Does menopausal hormone replacement therapy interact with known factors to increase risk of breast cancer? J Clin Oncol. 2002;20(3): 699-706.

67. Bernstein L, Pike MC, Ross RK, Judd HL, Brown JB, Henderson BE. Estrogen and sex hormone-binding globulin levels in nulliparous and parous women. J Natl Cancer Inst. 1985;74(4):741-5.

68. Garcia-Closas M, Herbstman J, Schiffman M, Glass A, Dorgan JF. Relationship between serum hormone concentrations, reproductive history, alcohol consumption and genetic polymorphisms in pre-menopausal women. Int J Cancer. 2002:102(2):172-8

69. Schmadeka R, Harmon BE, Singh M. Triple-negative breast carcinoma: current and emerging concepts. Am J Clin Pathol. 2014;141(4):462-77.

70. Mayer IA, Abramson VG, Lehmann BD, Pietenpol JA. New strategies for triple-negative breast cancer-deciphering the heterogeneity. Clin Cancer Res. 2014;20(4):782-90.

71. Roman M, Graff-Iversen S, Weiderpass E, Vangen S, Sakshaug S, Hofvind S, Ursin G. Postmenopausal hormone therapy and breast cancer prognostic characteristics: a linkage between nationwide registries. Cancer Epidem Biomar. 2016;25(11):1464-73.

\section{Submit your next manuscript to BioMed Central} and we will help you at every step:

- We accept pre-submission inquiries

- Our selector tool helps you to find the most relevant journal

- We provide round the clock customer support

- Convenient online submission

- Thorough peer review

- Inclusion in PubMed and all major indexing services

- Maximum visibility for your research

Submit your manuscript at www.biomedcentral.com/submit 